\title{
Molecular Docking Studies and ADME Prediction of Novel Isatin Analogs with Potent Anti-EGFR Activity
}

Swastika Ganguly* and Biplab Debnath

Department of Pharmaceutical Science and Technology, Birla Institute of Technology, Ranchi, India

\begin{abstract}
Molecular docking studies were performed on 144 newly designed isatin analogs by using Glide $v 5.0$ on the active site of five crystal structures of EGFR enzymes (PDB ID 2J5F, 2ITW, 2ITY, 2ITX and1M17) to study the binding mode of these analogs. Binding mode analysis of the compounds with the highest docking scores $(-8.31,-5.90,-7.16$, -6. 395 and -8. 14) was carried out and were compared with that of the co crystallized ligands DJK_3021_A, AFN ${ }_{941}$, irressa, AMP-PNP and AQ4 in the active sites of 2J5F, 2ITW, 2ITY, 2ITX and $1 \mathrm{M} 17$ respectively. ADME properties of all the newly designed isatin analogs 1-144 was calculated by Qik Prop v3. 0. All the designed compounds were found to exhibit lead like properties from the calculated ADME properties.
\end{abstract}

Keywords: Cancer; Isatin; Epidermal growth factor receptor (EGFR); Tyrosine kinase (TK); Docking; ADME

\section{Introduction}

Cancer is defined as a group of diseases characterized by uncontrolled growth, and the spread of abnormal cells which if left untreated may lead to death [1]. Cancer continues to be a major health problem worldwide and more than ten million new cancer cases occur annually, roughly half of which is prevalent in the developed countries, and the disease causes over six million deaths a year [2].

Till date chemotherapy has been the mainstay of cancer therapy. However the use of available chemotherapeutics is often limited mainly due to undesirable side effects which include bone marrow depression, alopecia, drug-induced cancer, hepatotoxicity, along with a limited choice of available anti-cancer drugs [3].

Angiogenesis involves the proliferation of endothelial cells (ECs) in response to specific growth stimuli such as vascular endothelial growth factor (VEGF) of basic fibroblast growth factor (bFGF). Each step of the process is controlled by these regulatory growth factors that stimulate or inhibit angiogenesis. However, these control mechanisms are often disordered in several pathologic diseases including cancer. The growth and maintenance of solid tumors are highly dependent on neovascularization and can be regulated by compounds that interfere with either the stimulation or proliferation of ECs [4].

Angiogenesis has been intensely investigated as an attractive cancer therapeutic target during the last decade as angiogenesis is the first rate-limiting step for tumor cells to metastasize and is also essential for cancer growth [1].

Some important receptors involved in angiogenesis have been identified, including vascular endothelial growth factor receptor (VEGFR), epidermal growth factor receptor (EGFR), and several others. These growth factor receptor kinases play important roles in the development, progression, aggressiveness, and metastasis of many solid tumors, such as non small cell lung cancer (NSCLC), head and neck cancers, and glioblastomas. Particularly, the involvement of the EGFR family of tyrosine kinases in cancer proliferation suggests that an inhibitor which blocks the tyrosine kinase activity of the entire EGFR family could have significant therapeutic potential [5]. It is a transmembrane receptor protein comprising of four homologs i. e. EGFR/ErbB1/HER1, HER2/ Neu/ErbB2, HER3/ErbB3 and HER4/ ErbB4.

The isatin pharmacophore has attracted, and still attracts, much attention from medicinal chemists because of its structural resemblance to various moieties present in vitamins, proteins and nucleic acids. Isatin moieties are of great importance in their biological as well as synthetic approach of medicinal chemistry. From worldwide reported literature, the various derivatives of isatin are known to possess a range of biological properties including antibacterial and antifungal [6-10], antiviral [11-13], anti-HIV [14,15], antiglycation [16], anticonvulsant and sedative-hypnotic [17,18], anti-inflammatory [19] activities. Various isatin derivatives have been reported to possess cytotoxic activity [20-23]. Thus isatin is a biologically validated starting point for the design and synthesis of chemical libraries directed at these targets [24].

In recent years, rational drug design has become prevalent widely in the pharmaceutical industry. This involves the use of computational methods which are simple, non-expensive and speed up the process of designing novel and potent molecules with desired biological activity. Docking is a rational approach to drug design which seeks to predict the structure and binding free energy of a ligand-receptor complex given only the structures of the free ligand and receptor [25]. The setup for a ligand docking approach requires the following components: A target protein structure with or without a bound ligand, the molecules of interest or a database containing existing or virtual compounds for the docking process, and a computational framework that allows the implementation of the desired docking and scoring procedures. Docking accuracy reflects an algorithm's ability to discover a conformation (pose) (http://poseview. zbh. uni-hamburg. de) and alignment of a ligand relative to a cognate protein that is close to that experimentally observed and to recognize the pose as correct. Scoring is the identification of the correct binding pose by its lowest energy value, and the ranking of protein-ligand complexes according to their binding affinities [26]. Molecular docking is often used in virtual screening methods [27] whereby large virtual libraries of compounds

*Corresponding author: Swastika Ganguly, Associate Professor, Department of Pharmaceutical Sciences and Technology, Birla Institute of Technology, Ranchi, India, Tel: 09431327042; E-mail: swastikaganguly@bitmesra.ac.in

Received May 24, 2014; Accepted July 25, 2014; Published July 28, 2014

Citation: Ganguly S, Debnath B (2014) Molecular Docking Studies and ADME Prediction of Novel Isatin Analogs with Potent Anti-EGFR Activity. Med chem 4 558-568. doi:10.4172/2161-0444.1000194

Copyright: @ 2014 Ganguly S, et al. This is an open-access article distributed under the terms of the Creative Commons Attribution License, which permits unrestricted use, distribution, and reproduction in any medium, provided the original author and source are credited. 
are reduced in size to a manageable subset, which, if successful, includes molecules with high binding affinities to a target receptor.

Previously, synthesis, cytotoxicity and docking studies of hydrazones and Schiff bases of isatin on the target VEGFR-2 have been reported [28]. In the present communication, we wish to report the docking studies of newly designed isatin analogs in to the active site of different crystal structures of the epidermal growth factor receptor (EGFR) kinase domain in complex (PDB ID 1M17, 2J5F, 2ITX, 2ITW and 2ITY respectively) by Glide v5. 0 . The results from this study will be useful in understanding the essential pharmacophoric features required for the further development of isatins as anticancer agents.

\section{Materials and Methods}

\section{Computational methods by Glide 5.0}

Docking study was performed for all the designed compounds $1-144$ by Glide v5. 0 [29] installed in a single machine running on a 3.4 GHz Pentium 4 processor with 1 GB RAM and 160 GB Hard Disk with Red Hat Linux Enterprise version 5. 0 as the operating system.

\section{Protein structure preparation}

The X-ray crystallographic structures of the EGFR proteins (PDB entry code 2J5F, 1M17, 2ITW, 2ITX and 2ITY) were obtained from Brookhaven Protein Data Bank (RCSB) $[29,30]$. The proteins were prepared using the Protein Preparation Wizard. Preprocessed bond orders were assigned, hydrogens were added, metals were treated, and water molecules were deleted. Energy was minimized (Impref minimization) using RMSD 0. $30^{\circ} \mathrm{A}$. The $3 \mathrm{D}$ diagrams of the ligands were drawn by using Maestro 8. 5 implemented in Schrödinger's suite. The ligands were then prepared and minimized by means of the OPLS_2005 force field [31,32] and the partial atomic charges were computed using the same. The ligand-docking was performed with Glide module in Schrodinger. The XP (Extra Precision) protocol implemented in Glide was employed for the docking studies.

\section{Ligand structure preparation}

All the compounds used in the docking study with Glide were built within maestro by using build module of Schrodinger Inc. These structures were geometry optimized by means of the Optimized Potentials for Liquid Simulations-2005 (OPLS 2005) force field with the steepest descent followed by truncated Newton conjugate gradient protocol. Partial atomic charges were computed using the OPLS_2005 force field.

\section{Validation of docking protocol}

The most suitable method of evaluating the accuracy of a docking procedure is to determine how intimately the lowest energy pose predicted by the scoring function resembles an experimental binding mode as determined by X-ray crystallography. In the present study, extra precision Glide docking procedure was validated by removing AQ4 (Erlotinib), DJK_3021_A , AMP-PNP, AFN ${ }_{941}$, Iressa from the binding site and re-docking it to the EGFR proteins (PDB ID:1M17, 2J5F, 2ITX, 2ITW and 2ITY ). We found a very good agreement between the localization of the inhibitors upon docking and from the crystal structures. The root mean square deviations (RMSD) between the predicted conformation and the observed X-raycrystallographic conformation ofcompound AQ4(Erlotinib), DJK_3021_A, AMP-PNP, AFN941, Irressa equaled $1.737 \mathrm{~A}^{\circ}, 1.005 \mathrm{~A}^{\circ}, 2.744 \mathrm{~A}^{\circ}, 2.931 \mathrm{~A}^{\circ}, 2.412 \mathrm{~A}^{\circ}$. This indicates the reliability of the docking methodin reproducing the experimentally observed binding mode for 1M17, 2J5F, 2ITX, 2ITW and 2ITY.

\section{Docking and scoring function}

All the conformers from the confgen-ligprep output were docked in the EGFR tyrosine kinase active site. All default parameters were used for extra precision docking. Glide extra precision mode was employed for the current docking study. Best poses were chosen for energy minimization during docking, a distance dependent dielectric constant of 2. 0 and maximum number of minimization step of 100 was used. The docking simulations (ligand receptor interactions) are scored using the Extra Precision (XP) mode which is implemented in GLIDE v5. 0 .

Finally, the minimized poses were rescored using Schrodinger's proprietary GlideScore scoring function.

In this docking method, the ligands are flexible and receptor is rigid except that the protein active site which has slight flexibility. To include receptor flexibility the ligands were docked into different grids generated for five protein conformations [33,34].

\section{ADME prediction}

ADME properties were calculated using Qikprop v3. 0 tool of Schrodinger software. It predicts both physicochemically significant descriptors and pharmacokinetically significant properties. QikProp provides ranges for comparing a exacting molecule's properties with those of $95 \%$ of known drugs. QikProp also flags 30 types of reactive functional groups that may cause false positives in hight throughput screening (HTS) assays. It also evaluates the suitability of analogs based on Lipinski's rule of five [35], which is essential to ensure druglike pharmacokinetic profile while using rational drug design. All the analogs were neutralized before being used by Qikprop.

\section{Results and Discussion}

\section{Docking studies}

A large number of EGFR crystal structures have been reported in the literature which have different conformations. In this work we have considered five crystal structures (PDB ID:1M17, 2J5F, 2ITX, 2ITW and 2ITY ) that are co-crystallized with inhibitors AQ4 (Erlotinib), DJK_3021_A , AMP-PNP, AFN941, Irressa respectively. Docking studies were performed using Glide v5. 0 on five high resolution crystal structures of EGFR enzyme to study the binding modes of quality and quantum interactions between differently substituted newly designed isatin analogs (Table 1) with the enzyme epidermal growth factor receptor (EGFR) kinase domain in complex (PDB ID 1M17, 2J5F, 2ITX, 2ITW and 2ITY) results of which are depicted in Table 2.

Docking studies were performed using Glide v5. 0 in the active sites of five high resolution crystal structures of EGFR enzyme in order to investigate the possible interactions between the designed isatin analogs and the active site of the epidermal growth factor receptor (EGFR) kinase and were compared with the binding mode of the known EGFR inhibitors EGFRTK- Erlotinib complex or [6, 7-bis(2-methoxy-ethoxy)quinazoline-4-yl]-(3-ethynylphenyl) amine (AQ4), N-[4-(3- bromo phenylamino) quinazolin-6-yl] acrylamide (DJK_3021_A) , EGFR inhibitor AFN ${ }_{941}$ EGFR inhibitor AMP-PNP and EGFR inhibitor Irressa respectivly. The X-ray structure of the enzymes (PDB ID: 1M17, 2J5F, 2ITW , 2ITX and 2ITY) bounded with AQ4(Erlotinib) , DJK_3021_A, AFN ${ }_{941}$, AMP-PNP and Irressa was taken from the protein data bank; (http://www. rcsb. org/pdb).

The reliability of the docking results was first checked by comparing the best docking poses obtained for the cocrystallized inhibitor with its bound conformation. This was done by removing each ligand from 


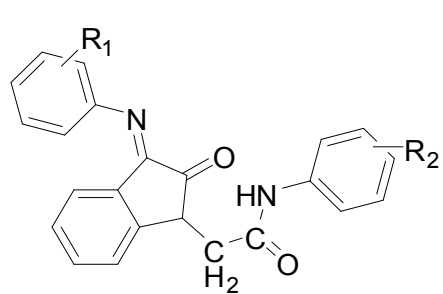

\begin{tabular}{|c|c|c|c|c|c|}
\hline Comp. Code & $R_{1}$ & $\mathbf{R}_{2}$ & Comp. Code & $\mathbf{R}_{1}$ & $\mathbf{R}_{2}$ \\
\hline 1 & $-\mathrm{H}$ & $-\mathrm{H}$ & 46 & $-4-\mathrm{Cl}$ & $2,5-\mathrm{CH}_{3}$ \\
\hline 2 & $-\mathrm{H}$ & $-2-\mathrm{Cl}$ & 47 & $-4-\mathrm{Cl}$ & $3,4-\mathrm{CH}_{3}$ \\
\hline 3 & $-H$ & $-3-\mathrm{Cl}$ & 48 & $-4-\mathrm{Cl}$ & $2-\mathrm{NO}_{2}$ \\
\hline 4 & $-\mathrm{H}$ & $-4-\mathrm{Cl}$ & 49 & $-2-\mathrm{CH}_{3}$ & $-H$ \\
\hline 5 & $-\mathrm{H}$ & $-2-\mathrm{CH}_{3}$ & 50 & $-2-\mathrm{CH}_{3}$ & $-2-\mathrm{Cl}$ \\
\hline 6 & $-\mathrm{H}$ & $-4-\mathrm{CH}_{3}$ & 51 & $-2-\mathrm{CH}_{3}$ & $-3-\mathrm{Cl}$ \\
\hline 7 & $-H$ & $3-\mathrm{OCH}_{3}$ & 52 & $-2-\mathrm{CH}_{3}$ & $-4-\mathrm{Cl}$ \\
\hline 8 & $-H$ & $4-\mathrm{OCH}_{3}$ & 53 & $-2-\mathrm{CH}_{3}$ & $-2-\mathrm{CH}_{3}$ \\
\hline 9 & $-\mathrm{H}$ & $2,4-\mathrm{CH}_{3}$ & 54 & $-2-\mathrm{CH}_{3}$ & $-4-\mathrm{CH}_{3}$ \\
\hline 10 & $-\mathrm{H}$ & $2,5-\mathrm{CH}_{3}$ & 55 & $-2-\mathrm{CH}_{3}$ & $3-\mathrm{OCH}_{3}$ \\
\hline 11 & $-\mathrm{H}$ & $3,4-\mathrm{CH}_{3}$ & 56 & $-2-\mathrm{CH} 3$ & $4-\mathrm{OCH}_{3}$ \\
\hline 12 & $-\mathrm{H}$ & $2-\mathrm{NO}_{2}$ & 57 & $-2-\mathrm{CH}_{3}$ & $2,4-\mathrm{CH}_{3}$ \\
\hline 13 & $-2-\mathrm{Cl}$ & $-\mathrm{H}$ & 58 & $-2-\mathrm{CH}_{3}$ & $2,5-\mathrm{CH}_{3}$ \\
\hline 14 & $-2-\mathrm{Cl}$ & $-2-\mathrm{Cl}$ & 59 & $-2-\mathrm{CH}_{3}$ & $3,4-\mathrm{CH}_{3}$ \\
\hline 15 & $-2-\mathrm{Cl}$ & $-3-\mathrm{Cl}$ & 60 & $-2-\mathrm{CH}_{3}$ & $2-\mathrm{NO}_{2}$ \\
\hline 16 & $-2-\mathrm{Cl}$ & $-4-\mathrm{Cl}$ & 61 & $-4-\mathrm{CH}_{3}$ & $-\mathrm{H}$ \\
\hline 17 & $-2-\mathrm{Cl}$ & $-2-\mathrm{CH}_{3}$ & 62 & $-4-\mathrm{CH}_{3}$ & $-2-\mathrm{Cl}$ \\
\hline 18 & $-2-\mathrm{Cl}$ & $-4-\mathrm{CH}_{3}$ & 63 & $-4-\mathrm{CH}_{3}$ & $-3-\mathrm{Cl}$ \\
\hline 19 & $-2-\mathrm{Cl}$ & $3-\mathrm{OCH}_{3}$ & 64 & $-4-\mathrm{CH}_{3}$ & $-4-\mathrm{Cl}$ \\
\hline 20 & $-2-\mathrm{Cl}$ & $4-\mathrm{OCH}_{3}$ & 65 & $-4-\mathrm{CH}_{3}$ & $-2-\mathrm{CH}_{3}$ \\
\hline 21 & $-2-\mathrm{Cl}$ & $2,4-\mathrm{CH}_{3}$ & 66 & $-4-\mathrm{CH}_{3}$ & $-4-\mathrm{CH}_{3}$ \\
\hline 22 & $-2-\mathrm{Cl}$ & $2,5-\mathrm{CH}_{3}$ & 67 & $-4-\mathrm{CH}_{3}$ & $3-\mathrm{OCH}_{3}$ \\
\hline 23 & $-2-\mathrm{Cl}$ & $3,4-\mathrm{CH}_{3}$ & 68 & $-4-\mathrm{CH}_{3}$ & $4-\mathrm{OCH}_{3}$ \\
\hline 24 & $-2-\mathrm{Cl}$ & $2-\mathrm{NO}_{2}$ & 69 & $-4-\mathrm{CH}_{3}$ & $2,4-\mathrm{CH}_{3}$ \\
\hline 25 & $-3-\mathrm{Cl}$ & $-\mathrm{H}$ & 70 & $-4-\mathrm{CH}_{3}$ & $2,5-\mathrm{CH}_{3}$ \\
\hline 26 & $-3-\mathrm{Cl}$ & $-2-\mathrm{Cl}$ & 71 & $-4-\mathrm{CH}_{3}$ & $3,4-\mathrm{CH}_{3}$ \\
\hline 27 & $-3-\mathrm{Cl}$ & $-3-\mathrm{Cl}$ & 72 & $-4-\mathrm{CH}_{3}$ & $2-\mathrm{NO}_{2}$ \\
\hline 28 & $-3-\mathrm{Cl}$ & $-4-\mathrm{Cl}$ & 73 & $3-\mathrm{OCH}_{3}$ & $-\mathrm{H}$ \\
\hline 29 & $-3-\mathrm{Cl}$ & $-2-\mathrm{CH}_{3}$ & 74 & $3-\mathrm{OCH}_{3}$ & $-2-\mathrm{Cl}$ \\
\hline 30 & $-3-\mathrm{Cl}$ & $-4-\mathrm{CH}_{3}$ & 75 & $3-\mathrm{OCH}_{3}$ & $-3-\mathrm{Cl}$ \\
\hline 31 & $-3-\mathrm{Cl}$ & $3-\mathrm{OCH}_{3}$ & 76 & $3-\mathrm{OCH}_{3}$ & $-4-\mathrm{Cl}$ \\
\hline 32 & $-3-\mathrm{Cl}$ & $4-\mathrm{OCH}_{3}$ & 77 & $3-\mathrm{OCH}_{3}$ & $-2-\mathrm{CH}_{3}$ \\
\hline 33 & $-3-\mathrm{Cl}$ & $2,4-\mathrm{CH}_{3}$ & 78 & $3-\mathrm{OCH}_{3}$ & $-4-\mathrm{CH}_{3}$ \\
\hline 34 & $-3-\mathrm{Cl}$ & $2,5-\mathrm{CH}_{3}$ & 79 & $3-\mathrm{OCH}_{3}$ & $3-\mathrm{OCH}_{3}$ \\
\hline 35 & $-3-\mathrm{Cl}$ & $3,4-\mathrm{CH}_{3}$ & 80 & $3-\mathrm{OCH}_{3}$ & $4-\mathrm{OCH}_{3}$ \\
\hline 36 & $-3-\mathrm{Cl}$ & $2-\mathrm{NO}_{2}$ & 81 & $3-\mathrm{OCH}_{3}$ & $2,4-\mathrm{CH}_{3}$ \\
\hline 37 & $-4-\mathrm{Cl}$ & $-H$ & 82 & $3-\mathrm{OCH}_{3}$ & $2,5-\mathrm{CH}_{3}$ \\
\hline 38 & $-4-\mathrm{Cl}$ & $-2-\mathrm{Cl}$ & 83 & $3-\mathrm{OCH}_{3}$ & $3,4-\mathrm{CH}_{3}$ \\
\hline 39 & $-4-\mathrm{Cl}$ & $-3-\mathrm{Cl}$ & 84 & $3-\mathrm{OCH}_{3}$ & $2-\mathrm{NO}_{2}$ \\
\hline 40 & $-4-\mathrm{Cl}$ & $-4-\mathrm{Cl}$ & 85 & $4-\mathrm{OCH}_{3}$ & $-\mathrm{H}$ \\
\hline 41 & $-4-\mathrm{Cl}$ & $-2-\mathrm{CH}_{3}$ & 86 & $4-\mathrm{OCH}_{3}$ & $-2-\mathrm{Cl}$ \\
\hline 42 & $-4-\mathrm{Cl}$ & $-4-\mathrm{CH}_{3}$ & 87 & $4-\mathrm{OCH}_{3}$ & $-3-\mathrm{Cl}$ \\
\hline 43 & $-4-\mathrm{Cl}$ & $3-\mathrm{OCH}_{3}$ & 88 & $4-\mathrm{OCH}_{3}$ & $-4-\mathrm{Cl}$ \\
\hline 44 & $-4-\mathrm{Cl}$ & $4-\mathrm{OCH}_{3}$ & 89 & $4-\mathrm{OCH}_{3}$ & $-2-\mathrm{CH}_{3}$ \\
\hline 45 & $-4-\mathrm{Cl}$ & $2,4-\mathrm{CH}_{3}$ & 90 & $4-\mathrm{OCH}_{3}$ & $-4-\mathrm{CH}_{3}$ \\
\hline 91 & $4-\mathrm{OCH}_{3}$ & $3-\mathrm{OCH}_{3}$ & 118 & $2,5-\mathrm{CH}_{3}$ & $2,5-\mathrm{CH}_{3}$ \\
\hline 92 & $4-\mathrm{OCH}_{3}$ & $4-\mathrm{OCH}_{3}$ & 119 & $2,5-\mathrm{CH}_{3}$ & $3,4-\mathrm{CH}_{3}$ \\
\hline 93 & $4-\mathrm{OCH}_{3}$ & $2,4-\mathrm{CH}_{3}$ & 120 & $2,5-\mathrm{CH}_{3}$ & $2-\mathrm{NO}_{2}$ \\
\hline 94 & $4-\mathrm{OCH}_{3}$ & $2,5-\mathrm{CH}_{3}$ & 121 & $3,4-\mathrm{CH}_{3}$ & $-\mathrm{H}$ \\
\hline 95 & $4-\mathrm{OCH}_{3}$ & $3,4-\mathrm{CH} 3$ & 122 & $3,4-\mathrm{CH}_{3}$ & $-2-\mathrm{Cl}$ \\
\hline 96 & $4-\mathrm{OCH}_{3}$ & $2-\mathrm{NO}_{2}$ & 123 & $3,4-\mathrm{CH}_{3}$ & $-3-\mathrm{Cl}$ \\
\hline 97 & $2,4-\mathrm{CH}_{3}$ & $-\mathrm{H}$ & 124 & $3,4-\mathrm{CH}_{3}$ & $-4-\mathrm{Cl}$ \\
\hline 98 & $2,4-\mathrm{CH}_{3}$ & $-2-\mathrm{Cl}$ & 125 & $3,4-\mathrm{CH}_{3}$ & $-2-\mathrm{CH}_{3}$ \\
\hline
\end{tabular}

\begin{tabular}{|c|c|c|c|c|c|}
\hline 99 & $2,4-\mathrm{CH}_{3}$ & $-3-\mathrm{Cl}$ & 126 & $3,4-\mathrm{CH}_{3}$ & $-4-\mathrm{CH}_{3}$ \\
\hline 100 & $2,4-\mathrm{CH}_{3}$ & $-4-\mathrm{Cl}$ & 127 & $3,4-\mathrm{CH}_{3}$ & $3-\mathrm{OCH}_{3}$ \\
\hline 101 & $2,4-\mathrm{CH}_{3}$ & $-2-\mathrm{CH}_{3}$ & 128 & $3,4-\mathrm{CH}_{3}$ & $4-\mathrm{OCH}_{3}$ \\
\hline 102 & $2,4-\mathrm{CH}_{3}$ & $-4-\mathrm{CH}_{3}$ & 129 & $3,4-\mathrm{CH}_{3}$ & $2,4-\mathrm{CH}_{3}$ \\
\hline 103 & $2,4-\mathrm{CH}_{3}$ & $3-\mathrm{OCH}_{3}$ & 130 & $3,4-\mathrm{CH}_{3}$ & $2,5-\mathrm{CH} 3$ \\
\hline 104 & $2,4-\mathrm{CH}_{3}$ & $4-\mathrm{OCH}_{3}$ & 131 & $3,4-\mathrm{CH}_{3}$ & $3,4-\mathrm{CH}_{3}$ \\
\hline 105 & $2,4-\mathrm{CH}_{3}$ & $2,4-\mathrm{CH}_{3}$ & 32 & $3,4-\mathrm{CH}_{3}$ & $2-\mathrm{NO}_{2}$ \\
\hline 106 & $2,4-\mathrm{CH}_{3}$ & $2,5-\mathrm{CH}_{3}$ & 133 & $2-\mathrm{NO}_{2}$ & $-H$ \\
\hline 107 & $2,4-\mathrm{CH}_{3}$ & $3,4-\mathrm{CH}_{3}$ & 134 & $2-\mathrm{NO}_{2}$ & $-2-\mathrm{Cl}$ \\
\hline 108 & $2,4-\mathrm{CH}_{3}$ & $2-\mathrm{NO}_{2}$ & 135 & $2-\mathrm{NO}_{2}$ & $-3-\mathrm{Cl}$ \\
\hline 109 & $2,5-\mathrm{CH}_{3}$ & $-\mathrm{H}$ & 136 & $2-\mathrm{NO}_{2}$ & $-4-\mathrm{Cl}$ \\
\hline 110 & $2,5-\mathrm{CH}_{3}$ & $-2-\mathrm{Cl}$ & 137 & $2-\mathrm{NO}_{2}$ & $-2-\mathrm{CH}_{3}$ \\
\hline 111 & $2,5-\mathrm{CH}_{3}$ & $-3-\mathrm{Cl}$ & 138 & $2-\mathrm{NO}_{2}$ & $-4-\mathrm{CH}_{3}$ \\
\hline 112 & $2,5-\mathrm{CH}_{3}$ & $-4-\mathrm{Cl}$ & 139 & $2-\mathrm{NO}_{2}$ & $3-\mathrm{OCH}_{3}$ \\
\hline 113 & $2,5-\mathrm{CH}_{3}$ & $-2-\mathrm{CH} 3$ & 140 & $2-\mathrm{NO}_{2}$ & $4-\mathrm{OCH} 3$ \\
\hline 114 & $2,5-\mathrm{CH}_{3}$ & $-4-\mathrm{CH}_{3}$ & 141 & $2-\mathrm{NO}_{2}$ & $2,4-\mathrm{CH}_{3}$ \\
\hline 115 & $2,5-\mathrm{CH}_{3}$ & $3-\mathrm{OCH} 3$ & 142 & $2-\mathrm{NO}_{2}$ & $2,5-\mathrm{CH}_{3}$ \\
\hline 116 & $2,5-\mathrm{CH}_{3}$ & $4-\mathrm{OCH}_{3}$ & 143 & $2-\mathrm{NO}_{2}$ & $3,4-\mathrm{CH}_{3}$ \\
\hline 117 & $2,5-\mathrm{CH}_{3}$ & $2,4-\mathrm{CH}_{3}$ & 144 & $2-\mathrm{NO}_{2}$ & $2-\mathrm{NO}_{2}$ \\
\hline
\end{tabular}

Table 1: Structures of newly designed isatin analogs 1-144.

\begin{tabular}{|c|c|c|c|c|c|c|}
\hline Compound & $\begin{array}{c}\text { Combined } \\
\text { (Gscore) }\end{array}$ & $\begin{array}{c}1 \mathrm{M} 17 \\
\text { (Gscore) }\end{array}$ & $\begin{array}{c}\text { 2J5F } \\
\text { (Gscore) }\end{array}$ & $\begin{array}{c}\text { 2ITW } \\
\text { (Gscore) }\end{array}$ & $\begin{array}{c}\text { 2ITX } \\
\text { (Gscore) }\end{array}$ & $\begin{array}{c}\text { 2ITY } \\
\text { (Gscore) }\end{array}$ \\
\hline Ref & -35.9 & -8.74 & -7.68 & -5.73 & -9.1 & -4.65 \\
\hline 143 & -32.29 & -8.10 & -5.88 & -5.66 & -4.98 & -7.67 \\
\hline 84 & -29.03 & -6.89 & -8.21 & -4.52 & -5.04 & -4.37 \\
\hline 120 & -28.88 & -8.09 & -5.91 & -4.98 & -4.76 & -5.14 \\
\hline 24 & -28.55 & -5.79 & -6.66 & -5.52 & -4.44 & -6.14 \\
\hline 139 & -28.5 & -5.43 & -7.26 & -5.07 & -3.33 & -7.41 \\
\hline 12 & -28.18 & -5.65 & -8.31 & -4.33 & -4.57 & -5.32 \\
\hline 108 & -28.1 & -8.14 & -5.62 & -4.51 & -4.92 & -4.91 \\
\hline 80 & -28.09 & -5.2 & -5.75 & -5.33 & -6.395 & -5.46 \\
\hline 62 & -28.08 & -7.6 & -6.26 & -4.87 & -5.18 & -4.17 \\
\hline 125 & -27.89 & -7.58 & -6.25 & -5.01 & -5.09 & -3.96 \\
\hline 144 & -27.89 & -5.15 & -6.45 & -4.72 & -4.69 & -6.88 \\
\hline 55 & -27.38 & -6.24 & -5.52 & -5.42 & -4.51 & -5.69 \\
\hline 137 & -27.37 & -4.17 & -7.65 & -4.34 & -4.32 & -6.89 \\
\hline 106 & -27.31 & -7.1 & -6.51 & -4.37 & -4.29 & -5.04 \\
\hline 73 & -27.25 & -5.79 & -5.65 & -5.19 & -5.67 & -4.95 \\
\hline 19 & -27.13 & -6.48 & -5.22 & -5.26 & -5.23 & -4.94 \\
\hline 142 & -27.90 & -8.05 & -4.49 & -4.68 & -4.88 & -4.99 \\
\hline 79 & -27.08 & -5.9 & -4.4 & -4.37 & -6.23 & -6.18 \\
\hline 132 & -27.01 & -6.1 & -6.3 & -4.78 & -4.61 & -5.22 \\
\hline 82 & -26.99 & -5.8 & -5.8 & -3.76 & -5.72 & -5.91 \\
\hline 96 & -26.85 & -5.93 & -6.07 & -4.21 & -5.8 & -4.84 \\
\hline 48 & -26.85 & -5.9 & -6.25 & -4.5 & -5.57 & -4.63 \\
\hline 95 & -26.75 & -5.84 & -6.06 & -3.88 & -5.78 & -5.19 \\
\hline N85 & -26.72 & -5.81 & -6.06 & -4.29 & -5.9 & -4.66 \\
\hline 41 & -26.68 & -6.03 & -6.23 & -4.04 & -5.48 & -4.9 \\
\hline 75 & -26.57 & -6.25 & -6.13 & -3.78 & -6.03 & -4.38 \\
\hline 77 & -26.57 & -5.85 & -5.85 & -4.18 & -5.92 & -4.77 \\
\hline 127 & -26.56 & -5.95 & -6.25 & -4.64 & -4.09 & -5.63 \\
\hline 32 & -26.44 & -5.83 & -4.41 & -5.05 & -5.92 & -5.23 \\
\hline 60 & -26.42 & -5.45 & -6.24 & -4.66 & -5.33 & -4.74 \\
\hline 20 & -26.4 & -5.63 & -5.53 & -5.24 & -5.5 & -4.5 \\
\hline 29 & -26.34 & -7.3 & -5.86 & -4.33 & -3.71 & -5.14 \\
\hline 8 & -26.34 & -5.68 & -5.81 & -5.01 & -4.55 & -5.29 \\
\hline 91 & -26.14 & -6.3 & -5.88 & -4.93 & -4.11 & -4.92 \\
\hline 130 & -26.14 & -6.17 & -6.54 & -3.51 & -4.88 & -5.04 \\
\hline 40 & -26.12 & -6.16 & -6.31 & -3.31 & -5.51 & -4.83 \\
\hline 123 & -26.08 & -6.4 & -6.24 & -4.52 & -3.55 & -5.37 \\
\hline
\end{tabular}




\begin{tabular}{|c|c|c|c|c|c|c|}
\hline Compound & $\begin{array}{l}\text { Combined } \\
\text { (Gscore) }\end{array}$ & $\begin{array}{c}\text { 1M17 } \\
\text { (Gscore) }\end{array}$ & $\begin{array}{c}2 \mathrm{~J} 5 \mathrm{~F} \\
\text { (Gscore) }\end{array}$ & $\begin{array}{c}\text { 2ITW } \\
\text { (Gscore) }\end{array}$ & $\begin{array}{c}\text { 2ITX } \\
\text { (Gscore) }\end{array}$ & $\begin{array}{c}\text { 2ITY } \\
\text { (Gscore) }\end{array}$ \\
\hline 83 & -26.06 & -6.45 & -5.43 & -4.49 & -4.62 & -5.07 \\
\hline N46 & -26.01 & -6.24 & -5.96 & -4.45 & -4.46 & -4.9 \\
\hline N38 & -26 & -6.48 & -6.24 & -4.12 & -4.56 & -4.6 \\
\hline 68 & -25.94 & -5.94 & -5.68 & -5.91 & -4.1 & -4.31 \\
\hline 141 & -25.91 & -8.37 & -5.79 & -4.01 & -5.09 & -2.65 \\
\hline 36 & -25.87 & -6.03 & -5.69 & -3.82 & -5.67 & -4.66 \\
\hline 140 & -25.87 & -8.21 & -5.41 & -5.7 & -3.67 & -2.88 \\
\hline 2 & -25.86 & -5.86 & -5.99 & -4.64 & -3.79 & -5.58 \\
\hline 121 & -25.75 & -6.63 & -4.14 & -5.05 & -4.62 & -5.31 \\
\hline 11 & -25.69 & -5.75 & -5.29 & -5.05 & -4.56 & -5.04 \\
\hline 78 & -25.66 & -5.97 & -4.78 & -4.59 & -5.74 & -4.58 \\
\hline 5 & -25.65 & -6.07 & -5.8 & -3.99 & -5.13 & -4.66 \\
\hline 76 & -25.61 & -5.74 & -5.84 & -3.99 & -5.08 & -4.96 \\
\hline 138 & -25.59 & -4.89 & -4.58 & -4.4 & -4.32 & -7.4 \\
\hline 44 & -25.57 & -5.94 & -4.56 & -4.8 & -5.96 & -4.31 \\
\hline 56 & -25.52 & -6.1 & -5.38 & -5.45 & -4.35 & -4.24 \\
\hline 14 & -25.49 & -5.64 & -5.26 & -4.55 & -4.76 & -5.28 \\
\hline 3 & -25.4 & -6.07 & -5.92 & -4.46 & -4.54 & -4.41 \\
\hline 70 & -25.36 & -5.61 & -6.03 & -3.95 & -5.21 & -4.56 \\
\hline 115 & -25.18 & -6.32 & -3.58 & -4.4 & -5.69 & -5.19 \\
\hline 97 & -25.16 & -5.54 & -5.47 & -4.88 & -3.78 & -5.49 \\
\hline 98 & -25.15 & -5.54 & -6.47 & -4.63 & -4.02 & -4.49 \\
\hline 74 & -25.02 & -6.13 & -5.57 & -3.71 & -4.96 & -4.65 \\
\hline 1 & -25.02 & -5.59 & -5.77 & -4.52 & -4.84 & -4.3 \\
\hline 45 & -24.96 & -5.9 & -6.22 & -4.46 & -3.59 & -4.79 \\
\hline 61 & -24.95 & -6.28 & -6.42 & -4.58 & -3.44 & -4.23 \\
\hline 10 & -24.92 & -5.45 & -4.67 & -4.21 & -5.26 & -5.33 \\
\hline 39 & -24.91 & -5.61 & -6.04 & -3.89 & -5.64 & -3.73 \\
\hline 28 & -24.82 & -5.79 & -4.18 & -4.83 & -4.92 & -5.1 \\
\hline 16 & -24.81 & -5.58 & -5.39 & -4.86 & -4.92 & -4.06 \\
\hline 72 & -24.8 & -5.06 & -4.96 & -4.73 & -5.77 & -4.28 \\
\hline 27 & -24.8 & -6.19 & -5.56 & -4.84 & -3.4 & -4.81 \\
\hline 9 & -24.79 & -5.94 & -5.12 & -4.61 & -4.98 & -4.14 \\
\hline 87 & -24.79 & -5.53 & -4.51 & -4.01 & -5.71 & -5.03 \\
\hline 53 & -24.73 & -5.43 & -6.03 & -4.43 & -3.94 & -4.9 \\
\hline 124 & -24.72 & -4.31 & -6.43 & -3.27 & -5.38 & -5.33 \\
\hline 47 & -24.71 & -3.46 & -6.12 & -4.28 & -5.58 & -5.27 \\
\hline 103 & -24.65 & -6.08 & -6.17 & -3.03 & -4.08 & -5.29 \\
\hline 111 & -24.49 & -5.54 & -4.33 & -5.08 & -5.09 & -4.45 \\
\hline 22 & -24.47 & -6.21 & -3.82 & -4.44 & -4.95 & -5.05 \\
\hline 23 & -24.44 & -5.31 & -4.47 & -4.61 & -4.74 & -5.31 \\
\hline Compound & $\begin{array}{l}\text { Combined } \\
\text { (Gscore) }\end{array}$ & $\begin{array}{c}1 \mathrm{M} 17 \\
\text { (Gscore) }\end{array}$ & $\begin{array}{c}\text { 2J5F } \\
\text { (Gscore) }\end{array}$ & $\begin{array}{c}21 T W \\
\text { (Gscore) }\end{array}$ & $\begin{array}{c}2 \mathrm{ITX} \\
\text { (Gscore) }\end{array}$ & $\begin{array}{c}\text { 2ITY } \\
\text { (Gscore) }\end{array}$ \\
\hline 135 & -24.34 & -5.03 & -4.66 & -4.85 & -4.79 & -5.01 \\
\hline 4 & -24.3 & -5.81 & -5.78 & -4.06 & -4.22 & -4.43 \\
\hline 86 & -24.27 & -3.15 & -6.3 & -4.81 & -4.64 & -5.37 \\
\hline 21 & -24.14 & -4.81 & -5.15 & -4.26 & -4.6 & -5.32 \\
\hline 31 & -24.1 & -6.26 & -3.64 & -4.36 & -4.24 & -5.6 \\
\hline 7 & -24.09 & -2.75 & -5.55 & -5.41 & -4.78 & -5.6 \\
\hline 65 & -24.04 & -7.19 & -6.27 & -4.04 & -2.22 & -4.32 \\
\hline 49 & -24.01 & -4.85 & -5.14 & -4.53 & -4.58 & -4.91 \\
\hline 101 & -23.97 & -5.76 & -4.16 & -4.22 & -4.62 & -5.21 \\
\hline 112 & -23.96 & -5.53 & -4.46 & -4.51 & -4.55 & -4.91 \\
\hline 51 & -23.95 & -5.45 & -4.5 & -5.09 & -4.23 & -4.68 \\
\hline 50 & -23.94 & -3.99 & -5.83 & -4.52 & -4.76 & -4.84 \\
\hline 67 & -23.83 & -4.38 & -4.97 & -4.82 & -4.31 & -5.35 \\
\hline 90 & -23.8 & -4.95 & -4.69 & -3.87 & -5.56 & -4.73 \\
\hline 107 & -23.74 & -4.72 & -4.06 & -4.48 & -4.7 & -5.78 \\
\hline 25 & -23.59 & -5.79 & -4.75 & -4.12 & -3.9 & -5.03 \\
\hline
\end{tabular}

\begin{tabular}{|c|c|c|c|c|c|c|}
\hline 58 & -23.59 & -4.86 & -5.56 & -4.05 & -4.28 & -4.84 \\
\hline 92 & -23.57 & -3.1 & -5.01 & -4.89 & -6.34 & -4.23 \\
\hline 89 & -23.49 & -6.12 & -4.3 & -4.15 & -3.81 & -5.11 \\
\hline 128 & -23.42 & -3.05 & -6.2 & -5.05 & -4.86 & -4.26 \\
\hline 129 & -23.38 & -3.36 & -6.85 & -3.9 & -4.55 & -4.72 \\
\hline 35 & -23.34 & -5.53 & -5.87 & -4.21 & -3.52 & -4.21 \\
\hline 30 & -23.07 & -6.19 & -4.97 & -2.56 & -4.93 & -4.42 \\
\hline 94 & -23.02 & -7.45 & -5.07 & -3.73 & -2.16 & -4.61 \\
\hline 119 & -22.97 & -5.1 & -5.93 & -3.44 & -4.55 & -3.95 \\
\hline 88 & -22.95 & -5.63 & -5.47 & -3.47 & -5.01 & -3.37 \\
\hline 93 & -22.93 & -5.52 & -3.97 & -3.73 & -5.32 & -4.39 \\
\hline 102 & -22.92 & -5.28 & -3.96 & -4.36 & -3.91 & -5.41 \\
\hline 43 & -22.9 & -5.71 & -4.14 & -4.75 & -4.5 & -3.8 \\
\hline 13 & -22.82 & -5.17 & -4.62 & -4.78 & -4.32 & -3.93 \\
\hline 116 & -22.79 & -5.21 & -4.62 & -4.85 & -3.39 & -4.72 \\
\hline 6 & -22.71 & -4.43 & -5.37 & -4.17 & -4.25 & -4.49 \\
\hline 33 & -22.62 & -3.65 & -5.91 & -3.67 & -5.09 & -4.3 \\
\hline 134 & -22.36 & -4.77 & -4.05 & -4.26 & -4.79 & -4.49 \\
\hline 109 & -22.29 & -5.67 & -4.5 & -4.32 & -3.54 & -4.26 \\
\hline 66 & -22.26 & -6.01 & -6.04 & -3.56 & -4.4 & -2.25 \\
\hline 131 & -22.25 & -3.7 & -3.89 & -4.26 & -5.69 & -4.71 \\
\hline 114 & -22.1 & -6.12 & -3.88 & -4.54 & -3.54 & -4.02 \\
\hline 118 & -22.04 & -5.85 & -4.15 & -4.48 & -3.87 & -3.69 \\
\hline 126 & -21.91 & -3.62 & -4.41 & -3.54 & -5.79 & -4.55 \\
\hline 26 & -21.86 & -6.27 & -5.89 & -4.03 & -4.05 & -1.62 \\
\hline Compound & $\begin{array}{c}\text { Combined } \\
\text { (Gscore) }\end{array}$ & $\begin{array}{c}1 \mathrm{M} 17 \\
\text { (Gscore) }\end{array}$ & $\begin{array}{c}\text { 2J5F } \\
\text { (Gscore) }\end{array}$ & $\begin{array}{c}2 I T W \\
\text { (Gscore) }\end{array}$ & $\begin{array}{c}\text { 2ITX } \\
\text { (Gscore) }\end{array}$ & $\begin{array}{c}\text { 2ITY } \\
\text { (Gscore) }\end{array}$ \\
\hline 113 & -21.74 & -4.24 & -4.91 & -4.27 & -3.65 & -4.67 \\
\hline 37 & -21.74 & -6.1 & -5.03 & -3.79 & -5.51 & -1.31 \\
\hline 133 & -21.64 & -4.59 & -4.29 & -4.22 & -4.71 & -3.83 \\
\hline 136 & -21.62 & -6.19 & -4.62 & -1.42 & -4.51 & -4.88 \\
\hline 15 & -21.56 & -4.68 & -4.08 & -4.85 & -4.4 & -3.55 \\
\hline 69 & -21.56 & -2.73 & -4.92 & -4.52 & -5.14 & -4.25 \\
\hline 59 & -21.32 & -1.75 & -4.14 & -4.34 & -5.43 & -5.66 \\
\hline 63 & -21.22 & -5.75 & -3.69 & -3.45 & -3.27 & -5.06 \\
\hline 110 & -21.16 & -2.95 & -4.36 & -4.55 & -4.95 & -4.35 \\
\hline 117 & -21.14 & -3.94 & -5.74 & -3.21 & -3.88 & -4.37 \\
\hline 17 & -21.08 & -4.09 & -4.46 & -4.46 & -4.37 & -3.7 \\
\hline 54 & -21 & -2.67 & -5.38 & -4.42 & -3.36 & -5.17 \\
\hline 81 & -20.92 & -2.85 & -4.22 & -3.9 & -5.18 & -4.77 \\
\hline 18 & -20.86 & -3.03 & -5.02 & -4.61 & -4.4 & -3.8 \\
\hline 34 & -20.54 & -2.58 & -5.5 & -3.95 & -3.62 & -4.89 \\
\hline 42 & -20.44 & -4.07 & -5.86 & -2.8 & -4.49 & -3.22 \\
\hline 57 & -20.43 & -4.76 & -5.55 & -2.14 & -3.9 & -4.08 \\
\hline 99 & -20.42 & -4.49 & -3.9 & -4.01 & -3.89 & -4.13 \\
\hline 105 & -20.18 & -5.12 & -5.87 & -4.3 & -4.2 & -0.69 \\
\hline 100 & -19.98 & -5.53 & -4.25 & -1.12 & -3.45 & -5.63 \\
\hline 64 & -18.83 & -3.52 & -3.95 & -3.85 & -3.53 & -3.98 \\
\hline 104 & -18.58 & -3.21 & -4.07 & -4.98 & -3.8 & -2.52 \\
\hline 71 & -17.71 & -2.41 & -6.25 & -3.85 & -3.2 & -2 \\
\hline 52 & -17.21 & -4.21 & -5.4 & -3.35 & -2.01 & -2.24 \\
\hline 122 & -15.79 & -5.06 & -6.18 & -4.96 & -4.38 & 4.79 \\
\hline
\end{tabular}

Table 2: Results of molecular docking studies of compounds 1-144 in the active sites of EGFR proteins (PDB ID1M17,2J5F,2ITW,2ITX and 2ITY) performed using extra precision mode of Glide.

their active site and subjecting again to redocking into the binding pocket in the conformation found in the crystal structure. As a result, a root mean square deviation (RMSD) of $1.737 \mathrm{~A}^{\circ}, 1.005 \mathrm{~A}^{\circ}, 2.744 \mathrm{~A}^{\circ}, 2$. $931 \mathrm{~A}^{\circ}, 2.412 \mathrm{~A}^{\circ}$ for EGFR proteins PDB ID:1M17, 2J5F, 2ITX, 2ITW and 2 ITY cocrystalized with. Erolotinib, DJK, AFN $_{941}, \mathrm{AMP}-\mathrm{PNP}$ and Irressa respectively were found suggesting that the docking procedure could be relied onto predict the binding mode of our compounds. 
The X-ray structure of the enzyme cocrystallized with DJK_3021_A was taken from the proteindata bank; PDB ID 2J5F [25]. The EGFR tyrosine kinase binding site contains the important residues $\mathrm{Thr} 790$, Met 793, Lys 745, Met 766, Cys797, Ala 743 and Leu788. The three dimensional docked pose of DJK_3021_A and the compound 12 in the active site of 2J5F has been depicted in Figure 1a and Figure 2a while the residues involved in inter-atomic contact has been shown in the schematic $2 \mathrm{D}$ representation as in Figure $1 \mathrm{~b}$ and Figure $2 \mathrm{~b}$ respectively. The binding mode analysis revealed that the the isatin scaffold in compound 12 is oriented in the binding site similarly as the quinazoline moiety of the cocrystallized ligand DJK_3021_A. The isatin scaffold is favorably embedded in the hydrophobic pocket surrounded by the side chains of Leu 718, Lys745 and Phe723. The compound also shows one $\mathrm{H}$-bond interaction between the hydrophilic spacer group $\mathrm{CH}_{2}-\mathrm{CO}-$ $\mathrm{NH}$ and the hydroxyl group present in residue ASP $855 \mathrm{NH}_{\mathrm{CH} 3 \mathrm{CHCONH}}$

$\mathrm{OH}_{\mathrm{ASP} 855}=1.643 \mathrm{~A}^{\circ}$ ). These interactions may be responsible for the binding affinity of the molecule as indicated by the docking scores -8 . 31 comparable and more than the docking score -7.68 of the reference ligand DJK_3021_A.

The 2ITW X ray crystal structure is co-crystallized with the ligand Staurosporine which has multiple ring structures and therefore is mostly stabilized by the hydrophobic interactions contributed by Leu718, Leu792, Leu844 and Lys745 while hydrogen bonds are present between the receptor residues Met793 and the ligand Staurosporine which is displayed in Figure 3a and 3b [36]. The docking pose of compound 68 in the active site of 2ITW has been represented in its three dimensional mode in Figure 4a while the schematic 2D dimensional representation has been shown in Figure 4b. The docking pose analysis revealed that the isatin scaffold is oriented in the hydrophobic pocket surrounded by the side chains of Leu 718, Leu 844 , Lys745 and Asp 745 in the active site of the EGFR protein 2ITW. The compound also shows three $\mathrm{H}$-bond interactions, one being between $\mathrm{NH}$ group of the $-\mathrm{CH}_{2} \mathrm{CONH}$ and the $\mathrm{C}=\mathrm{O}$ group present in residue Met $793\left(\mathrm{NH}_{\mathrm{CH} 2 \mathrm{CONH}}-\mathrm{C}=\mathrm{O}\right.$ Met793 $=1.889 \mathrm{~A}^{\circ}$ ), a second $\mathrm{H}$-bond between $\mathrm{C}=\mathrm{O}$ group of isatin and $\mathrm{NH}$ group present in residue Met $793\left(\mathrm{C}=\mathrm{O}_{\text {isatin ring }} \mathrm{NH}_{\text {Met793 }}=2.041\right.$ $\mathrm{A}^{\circ}$ ) and the third one being between the methoxy group substituted at the para position of the phenyl ring with the $\mathrm{NH}$ group present in the Lys 716 residue $\left.\left(\mathrm{OCH}_{3 \text { phenyl ring }-\mathrm{NH}}{ }_{\text {Lys } 716}=2.148 \mathrm{~A}^{\circ}\right)\right)$. These interactions increase the binding affinity of the molecule as indicated by the docking score of the compound 68 as -5.90 comparable and slightly more than the dock score -5.735 of the reference ligand Staurosporine.

The next EGFR protein (PDB ID 2ITY) cocrystallized with the ligand iressa shows only one $\mathrm{H}$-bond between the amide nitrogen of Met793 and the ligand as depicted in Figure 5a and 5b. In this case, the compound 79 , showed the highest docking score $(-7.16)$ in the active site of 2ITY [25]. In fact the dock score was higher than that of the cocrystallized ligand iressa $(-4.65)$. The three dimensional representation of the docked pose of compound 79 has been shown in Figure $6 \mathrm{a}$ and the residues involved in inter-atomic contact has been shown in Figure 6b. The docking pose study of compound 79 revealed that the isatin scaffold is oriented in the binding site similarly as in case

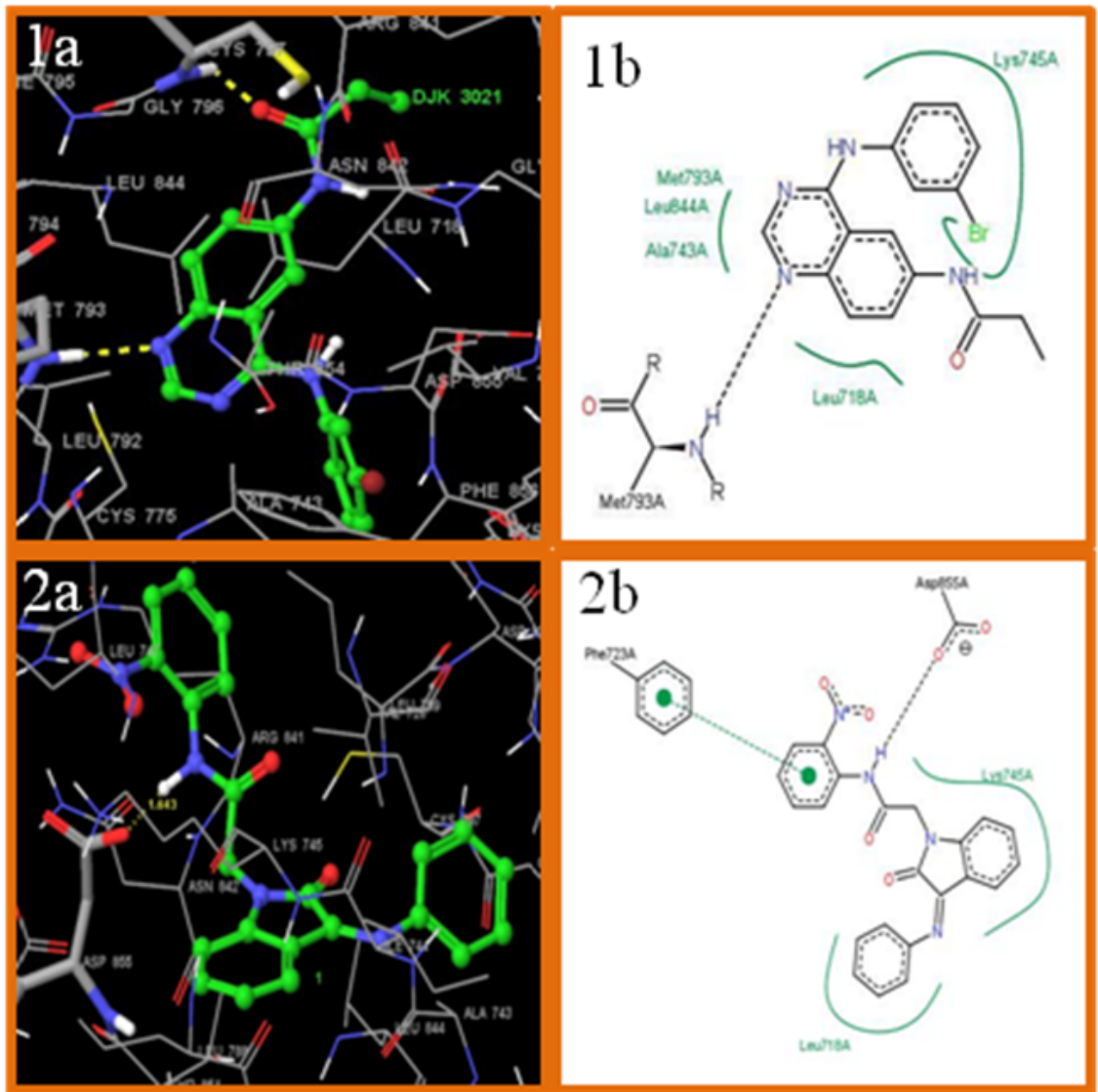

Figure 1(a). Redocked conformer of ligand DJK 3021_A in the active site of the protein EGFR (PDB ID 2J5F). 1(b). 2D representation of ligand DJK 3021 A. 2(a). Active site of the protein EGFR (PDB ID 2J5F) - molecular model compound 12. 2(b). Schematic 2D representation of interaction of compound 12 in the binding pocket of the protein. Active site amino acid residues are represented as tubes, while the inhibitor is shown as ball and stick model with the atoms colored as carbon: green, nitrogen: blue, oxygen: red. Hydrogen bond interactions are represented by yellow dotted lines. Pose view: black dashed lines - hydrogen bonds, salt bridges, metal interactions; green solid lines - hydrophobic interactions; green dashed lines - Pi-Pi, Pi-cation interaction. 

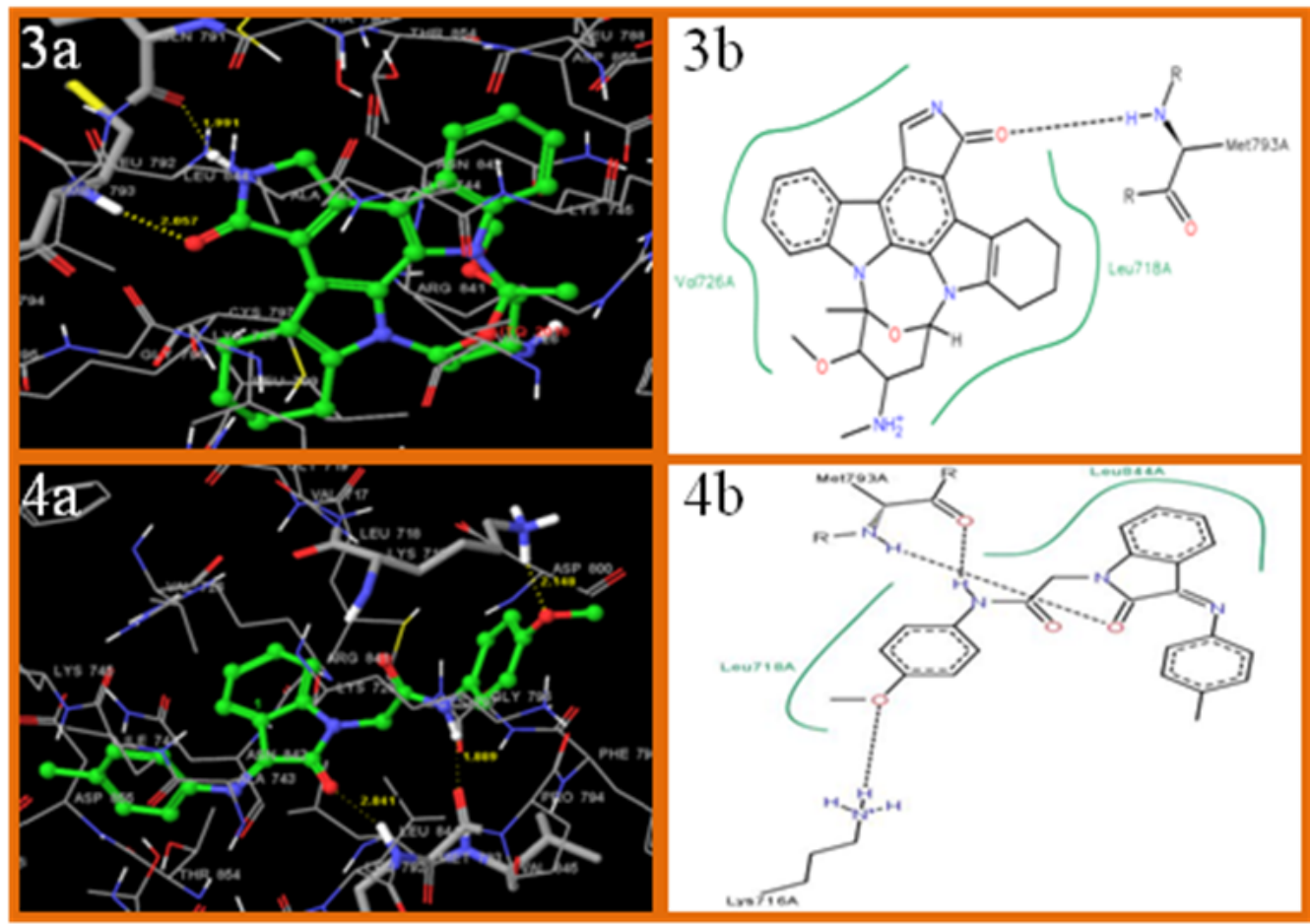

Figure 3(a). Redocked conformer of ligand $\mathrm{AFN}_{941}$ in active site of the protein EGFR (PDB ID 2ITW). 3(b). 2D representation of ligand AFN $\mathrm{A}_{941}$. $4(\mathrm{a})$. Active site of the protein EGFR (PDB ID 2ITW) of molecular model compound 68. 4(b). Schematic 2D representation of interactions of protein EGFR with compound 68in binding pocket. Active site amino acid residues are represented as tubes, while the inhibitor is shown as ball and stick model with the atoms colored as carbon: green, nitrogen: blue, oxygen: red. Hydrogen bond interactions are represented by yellow dotted lines. Pose view: black dashed lines - hydrogen bonds, salt bridges, metal interactions; green solid lines - hydrophobic interactions; green dashed lines - Pi-Pi, Pi-cation interaction.
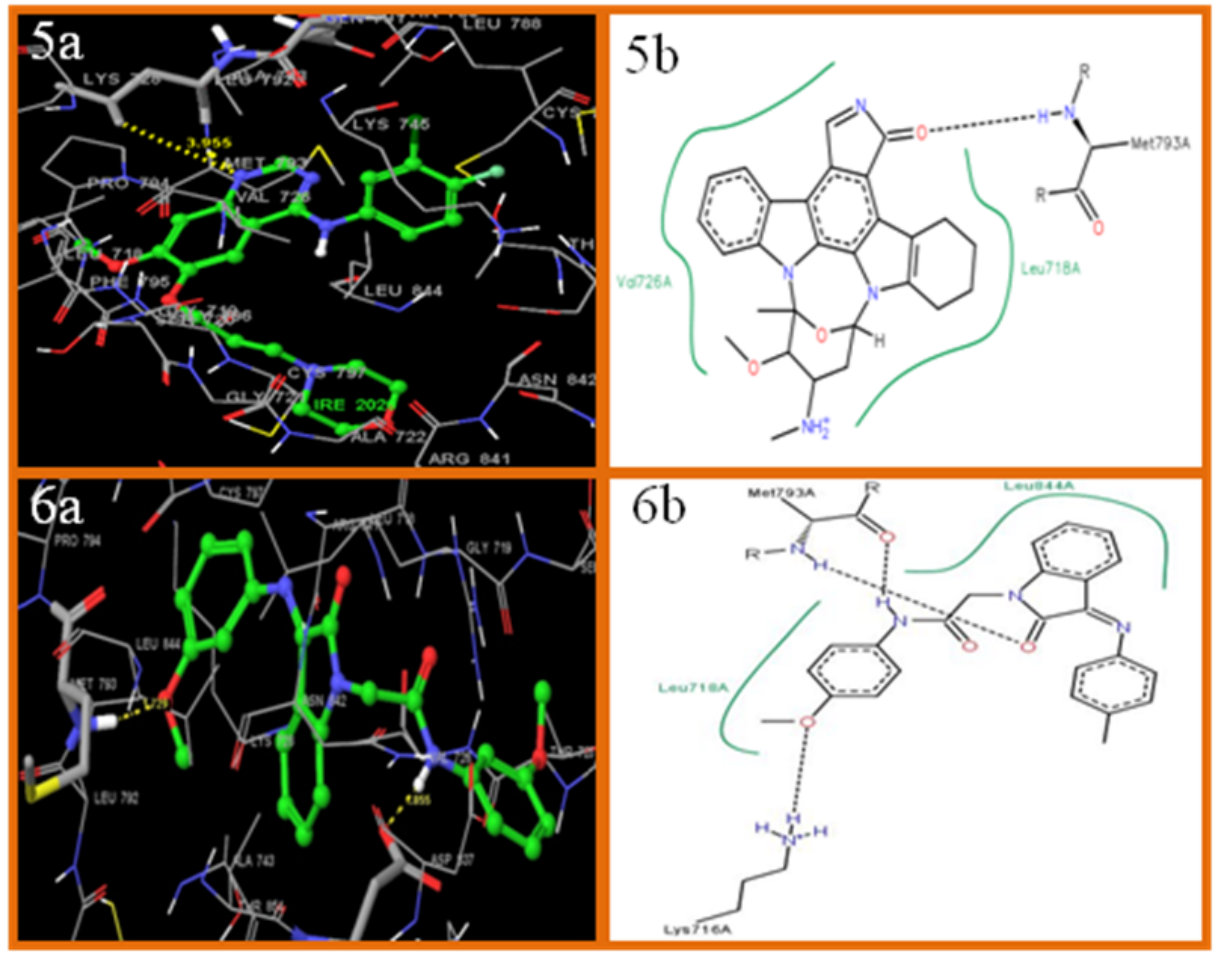

Figure 5(a). Redocked conformer of ligand irressa in active site of the protein EGFR (PDB ID 2ITY). 5(b). 2D representation of ligand irreessa. 6(a). Active site of the protein EGFR (PDB ID 2ITY) of molecular model compound 79. 6(b). Schematic 2D representation of interactions of compound 79 with protein EGFR in binding pocket. Active site amino acid residues are represented as tubes, while the inhibitor is shown as ball and stick model with the atoms colored as carbon: green, hydrogen: cyan, nitrogen: blue, oxygen: red. Hydrogen bond interactions are represented by yellow dotted lines.Pose view: black dashed lines - hydrogen bonds, salt bridges, metal interactions; green solid lines - hydrophobic interactions; green dashed lines - Pi-Pi, Pi-cation interaction. 
of the cocrystallized ligand Iressa in the active site of 2ITY. As in the previous cases, the isatin scaffold is oriented in the hydrophobic pocket surrounded by the side chains of Leu 844, Leu 718, Pro794, Lys745 and Phe723. The compound also shows two H-bond interactions between the $\mathrm{NH}$ group of the $-\mathrm{CH}_{2} \mathrm{CONH}$ and the hydroxyl group present in residue ASP $855\left(\mathrm{NH}_{\mathrm{CH} 3 \mathrm{CHCONH}} \_\mathrm{OH}=1.855 \mathrm{~A}^{\circ}\right)$ and between the methoxy group substituted at the meta position of the phenyl ring and $\mathrm{NH}$ goup present in residue Met $793\left(\mathrm{OCH}_{3 \text { Phenyl ring }} \mathrm{NH}_{\text {Met79 }}=1\right.$. $729 \mathrm{~A}^{\circ}$ ) respectively. These interactions and hydrogen bonding may increase the binding affinity of the molecule significantly as indicated by a very high docking score of of the compound 79 as compared to that of the cocrystallized ligand Irressa.

Further, the next EGFR protein (PDB ID 2ITX) co-crystallized with the ligand AMP-PNP also shows $\mathrm{H}$-bond interactions present between the receptor residues (Met793 and Asp855) and the cocrystallized ligand AMP-PNP as represented in Figure $7 \mathrm{a}$ and $7 \mathrm{~b}$ [25]. Among all the novel designed isatin analogs, compound 80 with the highest docking score in the active site of 2ITX is visualized in its three dimensional mode in Figure $8 \mathrm{a}$ and the residues involved in inter-atomic contact has been shown in Figure $8 \mathrm{~b}$. The docking pose visualization revealed that in compound 80 the isatin scaffold is oriented in the binding site similarly as the cocrystallized ligand AMP-PNP in the active site of 2ITX and is favorably embedded in the hydrophobic pocket surrounded by the side chains of Leu 844, Leu 718, Pro794, Lys745 and Phe723. The compound also shows two H-bond interactions, one between the $\mathrm{NH}$ group of the $-\mathrm{CH}_{2} \mathrm{CONH}$ and the $\mathrm{C}=\mathrm{O}$ group present in residue Pro794 $\left(\mathrm{NH}_{\mathrm{CH} 2 \mathrm{CONH}-\mathrm{CO}}{ }_{\text {Pro794 }}=2.138 \mathrm{~A}^{\circ}\right)$ and the second being between the $\mathrm{C}=\mathrm{O}$ group present in the isatin moiety linkage with $\mathrm{NH}$ group present in Met 793 residue $\left(\mathrm{CO}\right.$ isatin ring $\left.\mathrm{NH}_{\text {Met793 }}=2.038 \mathrm{~A}^{\circ}\right)$. However, the docking score of compound $80(-6.395)$ was less than that of the cocrystallized ligand AMP-PNP(-9. 101).

In case of PDB ID 1M17 complexed with the cocrystallized ligand erlotinib(AQ4), the ligand shows H-bond interactions with Met 769 as depicted in Figure 9a and 9b [37]. The interactions with threonine and methionine are very important for stable binding of AQ4 in the active site of $1 \mathrm{M} 17$. The three dimensional docked pose of compound 108 in the active site of 1M17 has been depicted in Figure 10a and the residues involved in inter-atomic contact has been shown in the schematic $2 \mathrm{D}$ representation as in Figure 10b. The docking pose study revealed that in compound 108 the isatin scaffold is oriented in the binding site likewise as the quinazoline moiety of erlotinib in the active site of $1 \mathrm{M} 17$. Here in, the isatin moiety interacts with multiple amino acid residues Met769, Leu820, Leu 764, Ala719, Lys721, Thr 766 , Thr 830 and Gly722. The compound also shows two H-bond interactions between the $\mathrm{C}=\mathrm{O}$ group of the $-\mathrm{CH}_{2} \mathrm{CONH}$ and the hydroxyl group present in residue Tyr $830\left(\mathrm{CO}_{\mathrm{CH} 3 \mathrm{CHCONH}}-\mathrm{OH}_{\mathrm{Tyr} 330}=2.250 \mathrm{~A}^{\circ}\right)$ while another hydrogen bond interaction was evident between oxygen atom of $\mathrm{NO}_{2}$ group at the para position of phenyl ring and hydrogen atom of $\mathrm{NH}$ group of Lys 712 residue $\left(\mathrm{NO}_{2 \text { phenyl ring_ }} \mathrm{NH}{ }_{\text {Lys } 712}=3.892 \mathrm{~A}^{\circ}\right)$. These interactions and the hydrogen bonding increases the binding affinity of the molecule as indicated by the docking scores -8.14 which is comparable to the dock score of the refernce ligand -8.745 .

\section{ADME properties}

We have analyzed 144 physically descriptors and pharmaceutically significant properties of isatin analogs using Qikprop v3. 0 tool of

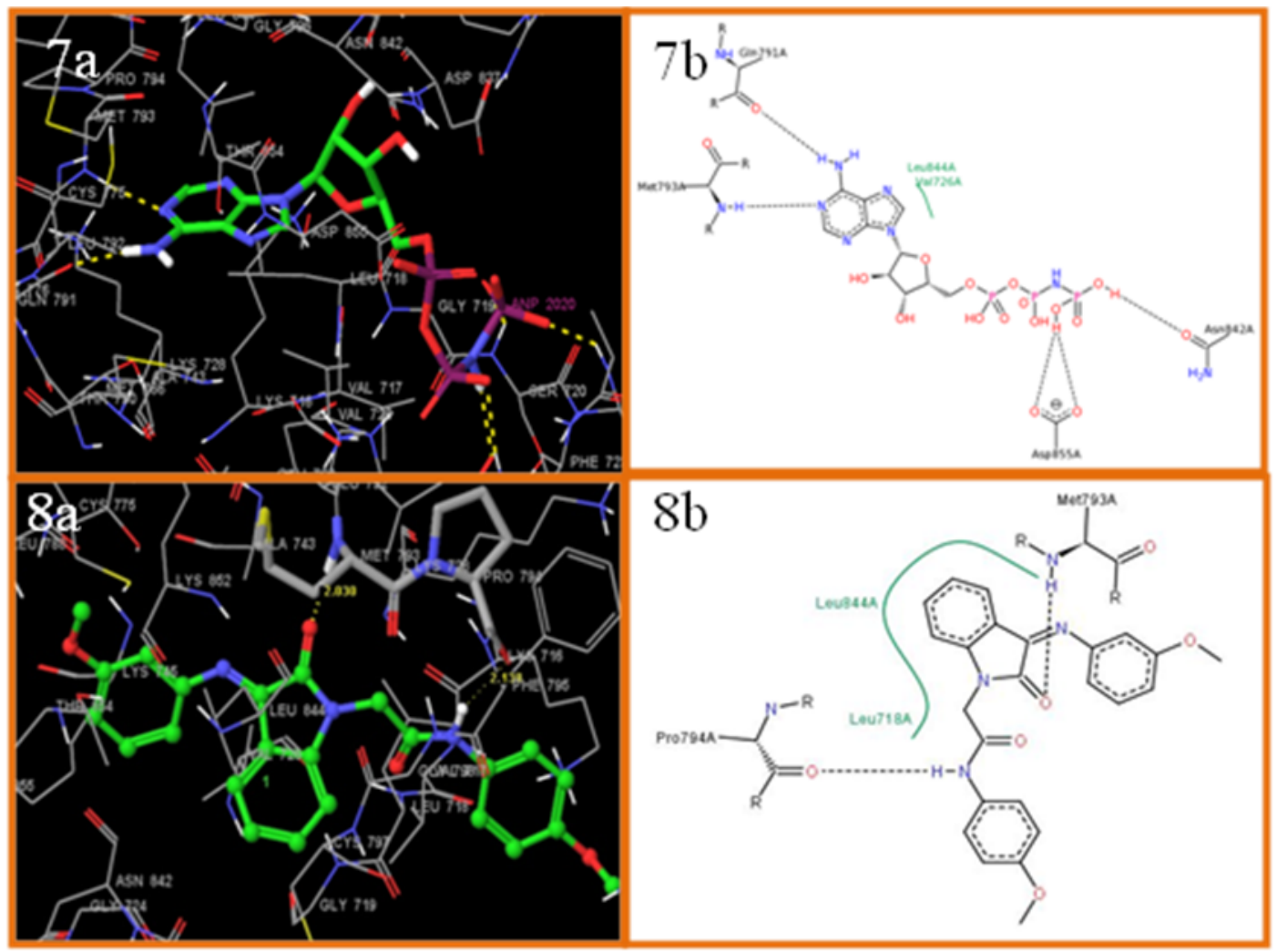

Figure 7(a). Redocked conformer of ligand AMP-PNP in active site of protein EGFR (PDB ID 2ITX). 7(b) 2D representation of ligand AMP-PNP. 8(a). Active site of the protein EGFR (PDB ID 2ITX) with Molecular model compound 80.8(b).Schematic 2D representation of interaction of compound 80 with protein EGFR in the binding pocket. Active site amino acid residues are represented as tubes, while the inhibitor is shown as ball and stick model with the atoms colored as carbon: green, hydrogen: cyan, nitrogen: blue, oxygen: red. Hydrogen bond interactions are represented by yellow dotted lines Pose view: black dashed lines - hydrogen bonds, salt bridges, metal interactions; green solid lines - hydrophobic interactions; green dashed lines - Pi-Pi, Pi-cation interaction. 


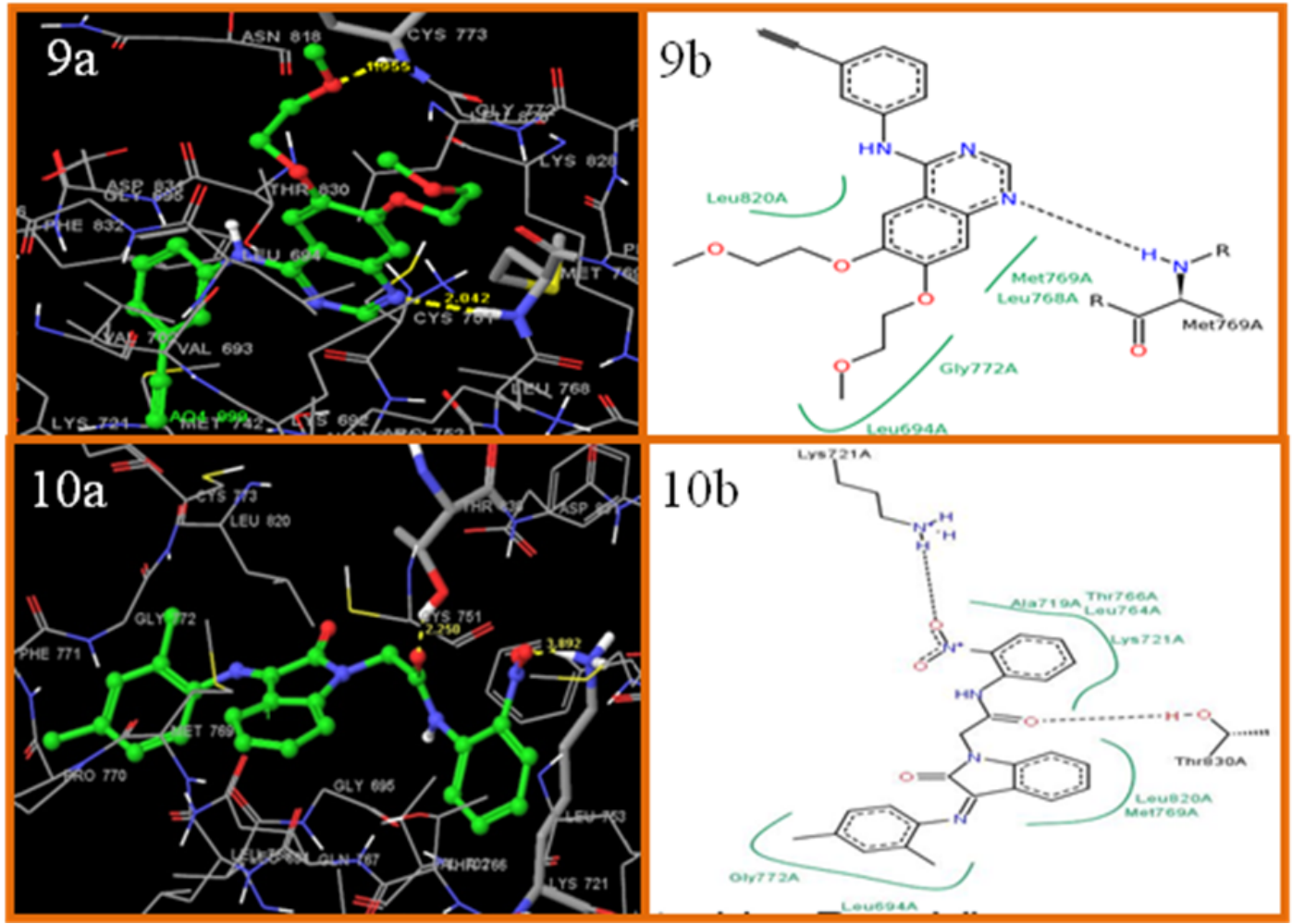

Figure 9(a). Redocked conformer of AQ4 in the active site of the protein EGFR (PDB ID 1M17), 9(b) 2D representation of ligand AQ4, 10(a).Active site of the protein EGFR (PDB ID 1M17) with molecular model compound 108 and 10(b). Schematic 2D representation of interaction of compound 108 with protein EGFR in the binding pocket. Active site amino acid residues are represented as tubes, while the inhibitor is shown as ball and stick model with the atoms colored as carbon: green, hydrogen: cyan, nitrogen: blue, oxygen: red. Hydrogen bond interactions are represented by yellow dotted lines Pose view: black dashed lines - hydrogen bonds, salt bridges, metal interactions; green solid lines - hydrophobic interactions; green dashed lines - $\mathrm{Pi}-\mathrm{Pi}, \mathrm{Pi}$-cation interaction.

Schrodinger software, among which major descriptors reported here are required for predicting the drug-like properties of molecules. These properties are

1. Molecular weight (mol_MW) (150-650)

2. Octanol/water partition coefficient ( $\log \mathrm{Po} / \mathrm{w})(-2-6.5)$

3. Aqueous solubility (QPlogS) (-6. 5-0.5)

4. Apparent MDCK cell permeability (QPPMDCK) ( $<25$ poor, $>500$ great)

5. Brain/blood partition coefficient (QPlogBB)(-3. 0-1.2)

6. Percent human oral absorption ( $\geq 80 \%$ is high, $\leq 25 \%$ is poor)

All the structures showed significant values for the properties analyzed (Table 3) and exhibited drug-like characteristics based on Lipinski's rule of 5 . The ADME values of newly designed compounds 1-144 are given in Table 3. The first three properties are based on Lipinski rule of five, molecular weight (mol_MW) less than 650, partition coefficient between octanol and water $(\log \mathrm{Po} / \mathrm{w})$ between -2 and 6. 5 and solubility (QPlogS) greater than -7. Brain/blood partition coefficient $(\mathrm{QP} \operatorname{logBB})$ parameter indicated about the ability of the drug to pass through the blood-brain barrier which is mandatory for inhibition of EGFR kinase. The QPPMDCK predicted apparent MDCK cell permeability in $\mathrm{nm} / \mathrm{s}$. MDCK cells are considered to be a good mimic for the blood-brain barrier. Higher the value of MDCK cell, higher the cell permeability.

All designed compounds showed ADME properties in acceptable range.

\section{Conclusion}

A number of newly designed isatin analogs 1-144 were docked into the active sites of five crystal structures of EGFR enzyme (PDB ID 2J5F, 2ITW, 2ITY, 2ITX and1M17) in order to investigate the possible interactions between the designed isatin analogs and the active site of the epidermal growth factor receptor (EGFR) kinase. The binding mode analysis of the compounds with the highest docking scores was carried out and were compared with that of the cocrystallized ligands DJK_3021_A, AFN ${ }_{941}$, irressa, AMP-PNP and AQ4 in the active sites of 2J5F, 2ITW, 2ITY, 2ITX and $1 \mathrm{M} 17$ respectively. It was found that compound 12 showed the highest docking score 8.31 in the active site of of the EGFR protein 2J5F. Compound 12 exhibited one hydrogen bond interaction and the dock score $(-8.31)$ was also higher than that of the reference standard $255 \mathrm{~F}$ (-7.665) while compound 68 , compound 79 , compound 80 and compound 108 showed highest docking score of $-5.90,-7.16,-6.395$ and -8.14 respectively in the active sites of EGFR proteins 2ITW, 2ITY, 2ITX and 1M17. Compound 68 exhibited three hydrogen bond interactions and the dock score $(-5.90)$ was also higher than that of the reference standard $\mathrm{AFN}_{941}(-5.735)$. However, compound 79 showed two hydrogen bond interactions with a dock score (-7.16) which was quite higher than that of the reference standard Irressa (-4.65). Compound 80 showed two hydrogen bond interactions, however the dock score (-6.395) was much lower than that of the reference standard AMP-PNP (-9.101). Compound 108 showed two hydrogen bond interactions and the dock score $(-8.14)$ was comparable to that of the reference standard AQ4 (-8.745). In all cases, the isatin moiety was oriented in a similar way as the reference ligand in the active sites of EGFR proteins 2J5F, 2ITW, 2ITY, 2ITX and 1 M17 respectively. It was observed from the docking results that all isatin analogs have a 


\begin{tabular}{|c|c|c|c|c|c|c|c|c|c|c|c|c|c|c|c|}
\hline \multirow{3}{*}{$\begin{array}{c}\text { Compound } \\
\text { code }\end{array}$} & \multirow{3}{*}{ Mol..Wt } & \multirow{3}{*}{ Log Po/w } & \multirow{3}{*}{$\log S$} & \multirow{3}{*}{ Log BB } & \multirow{3}{*}{ PMDCK } & \multirow{3}{*}{$\begin{array}{c}\text { Human } \\
\text { oral } \\
\begin{array}{c}\text { absorption } \\
(\%)\end{array} \\
\end{array}$} & \multirow{3}{*}{$\begin{array}{c}\text { Rule } \\
\text { of }\end{array}$} & 57 & 397.476 & 3.053 & \begin{tabular}{|l|}
-5.788 \\
\end{tabular} & -0.525 & 1552.5 & 100 & 0 \\
\hline & & & & & & & & 58 & 397.476 & 3.551 & -5.629 & -0.541 & 1544.5 & 100 & 0 \\
\hline & & & & & & & & 59 & 397.476 & 3.148 & -5.658 & -0.553 & 1550.5 & 100 & 0 \\
\hline 1 & 355.395 & 3.665 & -5.066 & -0.782 & 537.838 & 100 & 0 & 60 & 414.82 & 3.387 & -4.915 & -1.278 & 174.529 & 92.978 & 0 \\
\hline 2 & 389.84 & 4.181 & -5.382 & -0.433 & 1529.7 & 100 & 0 & 61 & 369.422 & 3.97 & -5.629 & -0.811 & 537.65 & 100 & 0 \\
\hline 3 & 389.84 & 4.451 & -5.995 & -0.378 & 2403 & 100 & 0 & 62 & 403.867 & 4.19 & -5.751 & -0.701 & 841.189 & 100 & 0 \\
\hline 4 & 389.84 & 4.451 & -5.992 & -0.377 & 2410.1 & 100 & 0 & 63 & 403.867 & 4.461 & -6.367 & -0.659 & 1322.9 & 100 & 0 \\
\hline 5 & 369.422 & 4.115 & -5.288 & -0.498 & 1008.6 & 100 & 0 & 64 & 403.867 & 4.461 & -6.364 & -0.657 & 1327 & 100 & 0 \\
\hline 6 & 369.422 & 4.268 & -5.827 & -0.558 & 976.111 & 100 & 0 & 65 & 383.449 & 4.124 & -5.658 & -0.766 & 554.74 & 100 & 0 \\
\hline 7 & 385.421 & 4.047 & -5.377 & -0.588 & 1016 & 100 & 0 & 66 & 383.449 & 4.276 & -6.196 & -0.84 & 537.619 & 100 & 0 \\
\hline 8 & 385.421 & 4.036 & -5.416 & -0.611 & 976.068 & 100 & 0 & 67 & 399.448 & 4.056 & -5.746 & -0.867 & 559.555 & 100 & 0 \\
\hline 9 & 383.449 & 4.592 & -6.218 & -0.49 & 1143.2 & 100 & 0 & 68 & 399.448 & 4.045 & -5.785 & -0.891 & 537.422 & 100 & 0 \\
\hline 10 & 383.449 & 4.591 & -6.218 & -0.491 & 1141.5 & 100 & 0 & 69 & 397.476 & 4.431 & -6.226 & -0.796 & 554.651 & 100 & 0 \\
\hline 11 & 383.449 & 4.535 & -6.238 & -0.0569 & 973.002 & 100 & 0 & 70 & 397.476 & 4.432 & -6.227 & -0.795 & 555.302 & 100 & 0 \\
\hline 12 & 400.393 & 3.388 & -4.758 & -1.1 & 276.456 & 96.294 & 0 & 71 & 397.476 & 4.546 & -6.414 & -0.855 & 535.698 & 100 & 0 \\
\hline 13 & 389.84 & 4.107 & -5.607 & 0.603 & 1233.9 & 100 & 0 & 72 & 414.42 & 3.395 & -5.122 & -1.37 & 152.419 & 92.053 & 0 \\
\hline 14 & 424.285 & 4.327 & -5.73 & -0.494 & 1930.2 & 100 & 0 & 73 & 385.421 & 3.744 & -5.232 & -0.863 & 537.865 & 100 & 0 \\
\hline 15 & 424.285 & 4.595 & -6.349 & -0.451 & 3028.9 & 100 & 0 & 74 & 419.866 & 3.964 & -5.355 & -0.753 & 841.508 & 100 & 0 \\
\hline 16 & 424.285 & 4.597 & -6.341 & -0.449 & 3045.7 & 100 & 0 & 75 & 419.866 & 4.234 & -5.967 & -0.711 & 1323.7 & 100 & 0 \\
\hline 17 & 403.867 & 4.183 & -5.637 & -0.56 & 1272.8 & 100 & 0 & 76 & 419.866 & 4.234 & -5.965 & -0.709 & 1327.5 & 100 & 0 \\
\hline 18 & 403.867 & 4.414 & -6.178 & -0.632 & 1230.6 & 100 & 0 & 77 & 399.448 & 3.899 & -5.261 & -0.819 & 554.993 & 100 & 0 \\
\hline 19 & 419.866 & 4.565 & -5.784 & -0.685 & 1230.9 & 100 & 0 & 78 & 399.488 & 4.049 & -5.795 & -0.892 & 537.842 & 100 & 0 \\
\hline 20 & 419.866 & 4.189 & -5.77 & -0.684 & 1230.2 & 100 & 0 & 79 & 430.419 & 3.835 & -5.386 & -0.925 & 559.748 & 100 & 0 \\
\hline 21 & 417.894 & 4.568 & -6.196 & -0.588 & 1272.66 & 100 & 0 & 80 & 430.419 & 3.817 & -5.384 & -0.943 & 537.664 & 100 & 0 \\
\hline 22 & 417.894 & 4.683 & -6.202 & -0.588 & 1272.4 & 100 & 0 & ind & Mol..Wt & Log Po/w & Log S & Log BB & PMDCK & $\begin{array}{c}\text { Human oral } \\
\text { absorption }\end{array}$ & $\begin{array}{l}\text { Rule } \\
\text { of }\end{array}$ \\
\hline 23 & 417.894 & 4.155 & -6.594 & -0.647 & 1226.6 & 100 & 0 & & & & & & & & five \\
\hline 24 & 434.838 & 3.47 & -5.663 & -1.529 & 179.204 & 88.394 & 0 & 81 & 413.475 & 4.373 & -6.187 & -0.82 & 629.597 & 100 & 0 \\
\hline 25 & 389.84 & 4.156 & -5.8 & -0.629 & 1326.9 & 100 & 0 & 82 & 413.475 & 4.373 & -6.189 & -0.827 & 628.756 & 100 & 0 \\
\hline 26 & 424.285 & 4.375 & -5.921 & -0.518 & 2076.2 & 100 & 0 & 83 & 413.475 & 4.317 & -6.811 & -0.831 & 627.953 & 100 & 0 \\
\hline 27 & 424.285 & 4.647 & -6.538 & -0.476 & 3266.1 & 100 & 0 & 84 & 430.419 & 3.169 & -4.724 & -1.419 & 152.493 & 90.733 & 0 \\
\hline 28 & 424.285 & 4.647 & -6.535 & -0.475 & 3275.8 & 100 & 0 & 85 & 385.421 & 3.747 & -5.248 & -0.865 & 537.6 & 100 & 0 \\
\hline 29 & 403.86 & 4.236 & -5.827 & -0.584 & 1368.3 & 100 & 0 & 86 & 419.866 & 3.967 & -5.371 & -0.756 & 841.247 & 100 & 0 \\
\hline 30 & 403.867 & 4.122 & -6.37 & -0.658 & 1326.8 & 100 & 0 & 87 & 419.866 & 4.238 & -5.985 & -0.713 & 1325.4 & 100 & 0 \\
\hline 31 & 419.866 & 3.889 & -5.959 & -0.691 & 1381.1 & 100 & 0 & 88 & 419.866 & 4.237 & -5.981 & -0.712 & 1327 & 100 & 0 \\
\hline 32 & 419.866 & 4.784 & -5.955 & -0.709 & 1325.6 & 100 & 0 & 89 & 399.488 & 4.071 & -5.639 & -0.801 & 629.366 & 100 & 0 \\
\hline 33 & 417.894 & 4.616 & -6.394 & -0.613 & 1368.2 & 100 & 0 & 90 & 399.488 & 4.052 & -5.811 & -0.894 & 537.621 & 100 & 0 \\
\hline 34 & 417.894 & 4.616 & -6.394 & -0.613 & 1369.6 & 100 & 0 & 91 & 415.488 & 3.825 & -5.413 & -0.947 & 537.631 & 100 & 0 \\
\hline 35 & 417.894 & 4.731 & -6.783 & -0.672 & 1322.4 & 100 & 0 & 92 & 415.488 & 3.82 & -5.4 & -0.946 & 537.329 & 100 & 0 \\
\hline 36 & 434.838 & 3.581 & -5.292 & -1.184 & 376.398 & 93.15 & 0 & 93 & 413.475 & 4.206 & -5.84 & -0.851 & 554.67 & 100 & 0 \\
\hline 37 & 389.84 & 4.646 & -5.799 & -0.628 & 1327.9 & 100 & 0 & 94 & 413.475 & 4.27 & -5.841 & -0.85 & 555.348 & 100 & 0 \\
\hline 38 & 424.285 & 4.648 & -6.353 & -0.405 & 3500.8 & 100 & 0 & 95 & 413.475 & 4.319 & -6.224 & -0.909 & 535.773 & 100 & 0 \\
\hline 39 & 424.285 & 3.581 & -6.538 & -0.476 & 3274.1 & 100 & 0 & 96 & 430.419 & 3.105 & -5.215 & -1.819 & 76.464 & 100 & 0 \\
\hline Compound & & & L $\mathrm{S}$ & $\mathrm{Rg} B$ & & Human oral & $\begin{array}{c}\text { Rule } \\
\text { of }\end{array}$ & 97 & 383.449 & 4.265 & -6.004 & -0.761 & 610.735 & 100 & 0 \\
\hline & Mol..Wt & $\log \mathrm{Po} / \mathrm{w}$ & $\log S$ & $\log B B$ & PMDCK & $\begin{array}{c}\text { absorption } \\
\text { (\%) }\end{array}$ & $\begin{array}{l}\text { of } \\
\text { five }\end{array}$ & 98 & 417.849 & 4.492 & -6.138 & -0.653 & 960.259 & 100 & 0 \\
\hline 40 & 424.285 & 4.632 & -6.534 & -0.475 & 3277.9 & 100 & 0 & 99 & 417.849 & 4.757 & -6.745 & -0.608 & 1503.2 & 100 & 0 \\
\hline 41 & 403.867 & 3.558 & -6.531 & -0.443 & 2317.8 & 100 & 0 & 100 & 417.849 & 4.757 & -6.742 & -0.607 & 1057.7 & 100 & 0 \\
\hline 42 & 403.867 & 4.552 & -6.365 & -0.657 & 1327.9 & 100 & 0 & 101 & 397.476 & 4.419 & -6.013 & -0.714 & 634.239 & 100 & 0 \\
\hline 43 & 419.866 & 4.248 & -5.969 & -0.696 & 1382.3 & 100 & 0 & 102 & 397.476 & 4.573 & -6.574 & -0.788 & 610.752 & 100 & 0 \\
\hline 44 & 419.866 & 4.231 & -5.956 & -0.709 & 1327.4 & 100 & 0 & 103 & 413.475 & 4.35 & -6.125 & -0.817 & 635.625 & 100 & 0 \\
\hline 45 & 417.894 & 4.787 & -6.76 & -0.591 & 1554.5 & 100 & 0 & 104 & 413.475 & 4.348 & -6.174 & -0.84 & 613.603 & 100 & 0 \\
\hline 46 & 417.894 & 4.787 & -6.76 & -0.592 & 1552.2 & 100 & 0 & 105 & 411.502 & 4.733 & -6.608 & -0.744 & 633.935 & 100 & 0 \\
\hline 47 & 417.894 & 4.731 & -6.782 & -0.672 & 1323.2 & 100 & 0 & 106 & 411.502 & 4.727 & -6.583 & -0.741 & 635.046 & 100 & 0 \\
\hline 48 & 434.834 & 3.581 & -5.293 & -1.184 & 376.405 & 93.147 & 0 & 107 & 411.502 & 4.846 & -6.983 & -0.797 & 613.802 & 100 & 0 \\
\hline 49 & 369.422 & 3.959 & -6.173 & -0.651 & 1547.9 & 100 & 0 & 108 & 428.446 & 3.689 & -5.493 & -1.33 & 173.048 & 94.684 & 0 \\
\hline 50 & 403.867 & 4.436 & -5.975 & -0.507 & 1618.8 & 100 & 0 & 109 & 383.449 & 4.26 & -6.003 & -0.766 & 604.261 & 100 & 0 \\
\hline 51 & 403.867 & 4.451 & -6.161 & -0.575 & 1507.8 & 100 & 0 & 110 & 417.849 & 4.743 & 6.723 & -0.531 & 1621 & 100 & 0 \\
\hline 52 & 403.867 & 4.45 & -6.174 & -0.579 & 174.529 & 100 & 0 & 111 & 417.849 & 4.753 & -6.745 & -0.612 & 1491.4 & 100 & 0 \\
\hline 53 & 383.449 & 4.114 & -5.45 & -0.686 & 634.361 & 100 & 0 & 112 & 417.849 & 4.752 & -6.741 & -0.612 & 1491.4 & 100 & 0 \\
\hline 54 & 383.449 & 4.265 & -5.988 & -0.756 & 614.087 & 100 & 0 & 113 & 397.476 & 4.591 & -6.337 & -0.69 & 719.916 & 100 & 0 \\
\hline 55 & 399.448 & 4.053 & -5.584 & -0.791 & 639.231 & 100 & 0 & 114 & 397.476 & 4.568 & -6.572 & -0.794 & 604.238 & 100 & 0 \\
\hline 56 & 399.448 & 4.034 & -5.579 & -0.609 & 613.9 & 100 & 0 & 115 & 413.475 & 4.338 & -6.14 & -0.816 & 639.997 & 100 & 0 \\
\hline
\end{tabular}




\begin{tabular}{|c|c|c|c|c|c|c|c|}
\hline 116 & 13.475 & 4.335 & -6.159 & -0.846 & 604.007 & 100 & 0 \\
\hline 117 & 11.502 & 4.727 & .576 & -0.74 & 635.166 & 100 & 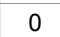 \\
\hline 118 & 11.502 & 4.728 & 6.577 & -0.739 & 35.976 & 100 & 0 \\
\hline 119 & 1.502 & 4.847 & 978 & -0.796 & 14.74 & 100 & 0 \\
\hline 120 & 8.446 & 683 & 491 & 1.334 & 1.268 & 94.578 & 0 \\
\hline 121 & 3.449 & 24 & 041 & -0.824 & 37.518 & 100 & 0 \\
\hline $\begin{array}{l}\text { Compound } \\
\text { code }\end{array}$ & Mol..Wt & Log Po/w & $\log S$ & $\log B B$ & PMDCK & $\begin{array}{l}\text { Human oral } \\
\text { absorption } \\
(\%)\end{array}$ & $\begin{array}{l}\text { Rule } \\
\text { of } \\
\text { five }\end{array}$ \\
\hline 122 & 417.849 & 4.459 & 163 & .715 & 1.028 & 100 & 0 \\
\hline 123 & 417.849 & 4.732 & .782 & -0.672 & 323 & 10 & 0 \\
\hline 124 & 7.849 & 4.732 & .779 & -0.67 & 326.8 & & 0 \\
\hline 125 & 397.476 & 4.566 & -6.436 & -0.759 & 629.303 & 10 & 0 \\
\hline 126 & 7.476 & 4.547 & -6.611 & -0.852 & 21 & 10 & 0 \\
\hline 127 & 3.475 & 332 & 2 & -0.886 & 73 & & 0 \\
\hline 128 & 3.475 & 4.314 & -6.196 & -0.904 & 37.353 & 100 & 0 \\
\hline 129 & 1.502 & 4.874 & -7.006 & -0.786 & 628.359 & 10 & 0 \\
\hline 130 & 1.502 & 4.874 & -7.008 & -0.868 & & & 0 \\
\hline 131 & 1.502 & 4.817 & 03 & 785 & 9.302 & 100 & 0 \\
\hline 132 & 8.446 & 3.663 & .529 & -1.392 & 152.406 & 10 & 0 \\
\hline 133 & 00.393 & 3.084 & -4.955 & -1.583 & 99.2 & 87.144 & 0 \\
\hline 134 & 4.838 & 3.32 & .161 & -1.481 & 151.165 & 88.074 & 0 \\
\hline 135 & 34.838 & 589 & -5.771 & -1.476 & 38.332 & 9.9 & 0 \\
\hline 136 & 434.838 & 3.588 & -5.766 & -1.475 & 38.614 & 9.9 & 0 \\
\hline 137 & 414.42 & 3.238 & -4.98 & -1.517 & 102.649 & 8.2 & 0 \\
\hline 138 & 4.42 & 3.402 & -5.596 & -1.662 & 96.633 & 96.6 & 0 \\
\hline 139 & 0.419 & 3.187 & -5.171 & -1.682 & 102.649 & 88 & 0 \\
\hline 140 & 430.419 & 156 & -5.105 & -1.678 & 99.192 & 87 & 0 \\
\hline 141 & 469.283 & 3.792 & -5.807 & -1.316 & 382.654 & 91.037 & 0 \\
\hline 142 & 469.283 & 3.792 & -5.806 & 1.316 & 382.464 & 1.0 & 0 \\
\hline 143 & 469.283 & 1 & -6.298 & -1.331 & 508.963 & 2.5 & 0 \\
\hline 144 & 445.39 & 2.511 & -4.45 & -2.103 & 28.011 & 61.734 & 1 \\
\hline AQ4 & 393.441 & 4.236 & -4.876 & -0.477 & 588.9 & 100 & 0 \\
\hline DJK & 371.236 & 3.585 & -5.168 & -0.331 & 2157.2 & 100 & 0 \\
\hline Irressa & 446.908 & 4.293 & -4.967 & -0.388 & 2646 & 100 & 0 \\
\hline AFN & 470.57 & 4.378 & -6.791 & -0.329 & 78.881 & 92.341 & 1 \\
\hline AMP-PNP & 506.20 & 5.771 & -6.753 & -0.386 & 784.228 & 100 & 0 \\
\hline
\end{tabular}

Table 3: Prediction of ADME properties of newly Designed isatin analogs using Qikprop.

common binding mode in the binding pockets of all the EGFR proteins. In all cases, hydrogen bonding interactions with the key residues were evident. ADME properties of all the newly designed compounds was studied by Qik Prop v3.0. All the designed compounds were found to exhibit lead like properties from the calculated ADME properties. These studies indicate that the newly designed isatin analogs may have a good binding affinity for EGFR enzyme. It can be concluded that the isatin moiety flanked by aryl rings substituted particularly with methyl, methoxy and nitro groups with a $\mathrm{CH}_{2} \mathrm{CONH}$ linker at the first position of the isatin ring structure may serve as a prominent scaffold for further synthesis of novel isatin analogs which could act as EGFR kinase inhibitors with promising anticancer activity.

\section{Acknowledgments}

The authors BD gratefully acknowledges the University Grants CommissionBasic Science Research (UGC-BSR) for the award of fellowship during the work.

\section{References}

1. Beale JM, Block J (2010) Organic medicinal and pharmaceutical chemistry. Lippincott Williams \& Wilkins.

2. Aboul-Fadl T, Radwan AA, Attia MI, Al-Dhfyan A, Abdel-Aziz HA (2012) Schiff bases of indoline-2,3-dione (isatin) with potential antiproliferative activity. Chem Cent J 6: 49.
3. Pourbasheer E, Amanlou M (2014) 3D-QSAR analysis of anti-cancer agents by CoMFA and CoMSIA. Medicinal Chemistry Research 23: 800-809.

4. Qian Y, Zhang HJ, Zhang H, Xu C, Zhao J, et al. (2010) Synthesis, molecular modeling, and biological evaluation of cinnamic acid metronidazole ester derivatives as novel anticancer agents. Bioorganic \& medicinal chemistry 18: 4991-4996.

5. Mendelsohn J, Baselga J (2000) The EGF receptor family as targets for cancer therapy. Oncogene 19: 6550-6565.

6. Pandeya SN, Sriram D, Nath G, DeClercq E (1999) Synthesis, antibacterial, antifungal and anti-HIV activities of Schiff and Mannich bases derived from isatin derivatives and $\mathrm{N}$-[4-(4'-chlorophenyl)thiazol-2-yl] thiosemicarbazide. Eur J Pharm Sci 9: 25-31.

7. Pandeya SN, Sriram D, Nath G, De Clercq E (2000) Synthesis, antibacterial, antifungal and anti-HIV activities of norfloxacin mannich bases. Eur $\mathrm{J}$ Med Chem 35: 249-255.

8. Sarangapani MR, Reddy VM (1994) Pharmacological evaluation of 1-(N $\mathrm{N}$-disubstituted aminomethyl)-3-imino-(2-phenyl-3, 4-dihydro- 4-oxoquinazolin-3-yl) indolin-2-ones. Indian J Pharm Sci 56, 174-177.

9. Varma RS, Nobles WL (1975) Antiviral, antibacterial, and antifungal activities of isatin N-Mannich bases. J Pharm Sci 64: 881-882.

10. Sridhar SK, Saravanan M, Ramesh A (2001) Synthesis and antibacterial screening of hydrazones, Schiff and Mannich bases of isatin derivatives. Eur J Med Chem 36: 615-625.

11. Logan JC, Fox MP, Morgan JH, Makohon AM, Pfau CJ (1975) Arenavirus inactivation on contact with $\mathrm{N}$-substituted isatin beta-thiosemicarbazones and certain cations. J Gen Virol 28: 271-283.

12. Varma RS, Nobles WL (1967) Synthesis and antiviral and antibacterial activity of certain $\mathrm{N}$-dialkylaminomethylisatin beta-thiosemicarbazones. J Med Chem 10: $972-974$

13. Singh SP, Shukla SK, Awasthi LP (1983) Synthesis of some 3-(40-nitrobenzoylhydrazone)-2-indolinones as potential antiviral agents. Curr Sci 52: 766-769.

14. Pandeya SN, Sriram D, Nath G, De Clercq E (1999) Synthesis, antibacterial, antifungal and anti-HIV evaluation of Schiff and Mannich bases of isatin derivatives with 3-amino-2-methylmercapto quinazolin-4(3H)-one. Pharm Acta Helv 74: 11-17.

15. Pandeya SN, Yogeeswari P, Sriram D, de Clercq E, Pannecouque C, et al. (1999) Synthesis and screening for anti-HIV activity of some N-Mannich bases of isatin derivatives. Chemotherapy 45: 192-196.

16. Khan KM, Khan M, Ali M, Taha M, Rasheed S, et al. (2009) Synthesis of bisSchiff bases of isatins and their antiglycation activity. Bioorg Med Chem 17 7795-7801.

17. Verma M, Pandeya SN, Singh KN, Stables JP (2004) Anticonvulsant activity of Schiff bases of isatin derivatives. Acta Pharm 54: 49-56.

18. Pandeya SN, Smitha S, Stables JP (2002) Anticonvulsant and sedativehypnotic activities of $\mathrm{N}$-substituted isatin semicarbazones. Arch Pharm (Weinheim) 335: 129-134.

19. Bhattacharya SK, Chakrabarti A (1998) Dose-related proconvulsant and anticonvulsant activity of isatin, a putative biological factor, in rats. Indian J Exp Biol 36: 118-121.

20. Matesic L, Locke JM, Bremner JB, Pyne SG, Skropeta D, et al. (2008) $\mathrm{N}$-phenethyl and $\mathrm{N}$-naphthylmethyl isatins and analogues as in vitro cytotoxic agents. Bioorg Med Chem 16: 3118-3124.

21. Hossain MM, Islam N, Khan R, Islam M (2008) Cytotoxicity study of dimethylisatin and its heterocyclic derivatives. Bangladesh $\mathrm{J}$ Pharmacol 2: 66-70.

22. Vine KL, Locke JM, Ranson M, Benkendorff K, Pyne SG, et al. (2007) In vitro cytotoxicity evaluation of some substituted isatin derivatives. Bioorg Med Chem 15: $931-938$

23. Pervez H, Ramzan M, Yaqub M, Mohammed Khan K (2011) Synthesis cytotoxic and phytotoxic effects of some new N4-aryl substituted isatin-3thiosemicarbazones. Lett Drug Des Discov 8: 452-458.

24. Shuttleworth SJ, Nasturica D, Gervais C, Siddiqui MA, Rando RF, et al. (2000) Parallel synthesis of isatin-based serine protease inhibitors. Bioorg Med Chem Lett 10: 2501-2504. 
Citation: Ganguly S, Debnath B (2014) Molecular Docking Studies and ADME Prediction of Novel Isatin Analogs with Potent Anti-EGFR Activity. Med chem 4: 558-568. doi:10.4172/2161-0444.1000194

25. Jones G, Willett $P$ (1995) Docking small-molecule ligands into active sites. Curr Opin Biotechnol 6: 652-656.

26. Muegge I, Rarey M (2001) Small molecule docking and scoring. Reviews in computational chemistry 17: 1-60.

27. Krovat EM, Steindl T, Langer T (2005) Recent advances in docking and scoring. Current Computer-Aided Drug Design 10: 93-102.

28. Azizian J, Mohammadi MK, Firuzi O, Razzaghi-asI N, Miri, R (2012) Synthesis, biological activity and docking study of some new isatin Schiff base derivatives. Medicinal Chemistry Research 2: 3730-3740.

29. Friesner RA, Banks JL, Murphy RB, Halgren TA, Klicic JJ, et al. (2004) Glide: a new approach for rapid, accurate docking and scoring. 1. Method and assessment of docking accuracy. J Med Chem 47: 1739-1749.

30. Berman HM, Battistuz T, Bhat TN, Bluhm WF, Bourne PE, et al. (2002) The Protein Data Bank. Acta Crystallogr D Biol Crystallogr 58: 899-907.

31. Hopkins AL, Ren J, Esnouf RM, Willcox BE, Jones EY, et al. (1996) Complexes of HIV-1 reverse transcriptase with inhibitors of the HEPT series reveal conformational changes relevant to the design of potent non-nucleoside inhibitors. J Med Chem 39: 1589-1600.
32. Jorgensen WL, Maxwell DS, Tirado-Rives J (1996) Development and testing of the OPLS all-atom force field on conformational energetics and properties of organic liquids. J Am Chem Soc 118: 11225-11236.

33. Carlson HA, Masukawa KM, McCammon JA (1999) Method for including the dynamic fluctuations of a protein in computer-aided drug design. J Phys Chem A, 103: 10213-10219.

34. Carlson HA, Masukawa KM, Rubins K, Bushman FD, Jorgensen WL, et al. (2000) Developing a dynamic pharmacophore model for HIV-1 integrase. J Med Chem 43: 2100-2114.

35. Lipinski CA, Lombardo F, Dominy BW, Feeney PJ (2001) Experimental and computational approaches to estimate solubility and permeability in drug discovery and development settings. Adv Drug Deliv Rev 46: 3-26.

36. Blair JA, Rauh D, Kung C, Yun CH, Fan QW, et al. (2007) Structure-guided development of affinity probes for tyrosine kinases using chemical genetics. Nat Chem Biol 3: 229-238.

37. Stamos J, Sliwkowski MX, Eigenbrot C (2002) Structure of the epidermal growth factor receptor kinase domain alone and in complex with a 4-anilinoquinazoline inhibitor. J Biol Chem 277: 46265-46272. 